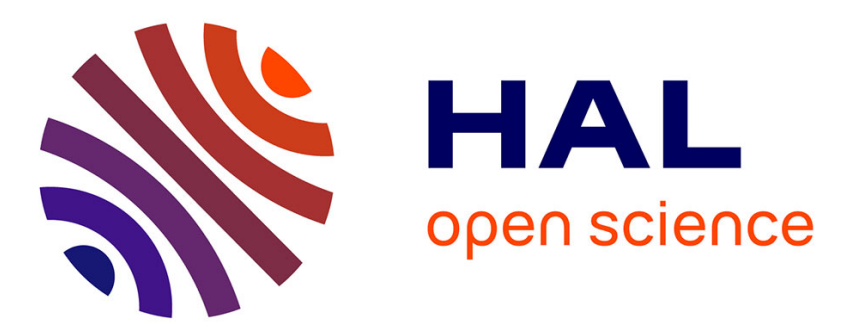

\title{
Barrier option hedging under constraints: a viscosity approach
}

\author{
Bruno Bouchard, Imen Bentahar
}

\section{To cite this version:}

Bruno Bouchard, Imen Bentahar. Barrier option hedging under constraints: a viscosity approach. SIAM Journal on Control and Optimization, 2008, 47 (4), pp.1785-1813. hal-00019886

\section{HAL Id: hal-00019886 https://hal.science/hal-00019886}

Submitted on 28 Feb 2006

HAL is a multi-disciplinary open access archive for the deposit and dissemination of scientific research documents, whether they are published or not. The documents may come from teaching and research institutions in France or abroad, or from public or private research centers.
L'archive ouverte pluridisciplinaire HAL, est destinée au dépôt et à la diffusion de documents scientifiques de niveau recherche, publiés ou non, émanant des établissements d'enseignement et de recherche français ou étrangers, des laboratoires publics ou privés. 


\title{
Barrier option hedging under constraints: a viscosity approach
}

\author{
Imen Bentahar* \\ TU Berlin, Germany \\ bentahar@math.tu-berlin.de
}

\author{
Bruno Bouchard \\ Université Paris VI, LPMA, and CREST \\ Paris, France \\ bouchard@ccr.jussieu.fr
}

February 28, 2006

\begin{abstract}
We study the problem of finding the minimal initial capital needed in order to hedge without risk a barrier option when the vector of proportions of wealth invested in each risky asset is constraint to lie in a closed convex domain. In the context of a Brownian diffusion model, we provide a PDE characterization of the super-hedging price. This extends the result of Broadie, Cvitanic and Soner (1998) and Cvitanic, Pham and Touzi (1999) which was obtained for plain vanilla options, and provides a natural numerical procedure for computing the corresponding super-hedging price. As a by-product, we obtain a comparison theorem for a class of parabolic PDE with relaxed Dirichet conditions involving a constraint on the gradient.
\end{abstract}

Key words : Super-replication, barrier options, portfolio constraints, viscosity solutions.

MSC Classification (2000): 91B28, 49L25, 35B05.

${ }^{*}$ This work was partly supported by the Deutsche Forschungsgemeinschaft through the SFB 649 "Economic Risk" 


\section{Introduction}

The problem of super-hedging under portfolio constraints has attracted a lot of attention since the seminal work of Cvitanic and Karatzas [5]. One of the original motivations came from the hedging of plain vanilla options with discontinuous payoffs, such as digital options. For such options the delta and gamma may take very large values when the remaining maturity is small, which makes them difficult to delta-hedge.

Within diffusion models, the remarkable result of Broadie, Cvitanic̀ and Soner [3] shows that the optimal hedge under constraints is obtained by considering the Black-Scholes type hedging strategy of some modified payoff. Thus, hedging the original claim under constraints corresponds to hedging a modified one without constraints. This is the socalled 'face-lifting' procedure. Within the Black-Scholes model, this allows to explicit the optimal hedge. In more general Markov diffusion models, an explicit solution may not be available but the super-hedging price can still be characterized as the solution of some Hamilton-Jacobi-Bellman equation, see Cvitanic̀, Pham and Touzi [6] and the review paper Soner and Touzi [12]. In the general semi-martingale case, no explicit solution is available but a general dual formulation was obtained by Föllmer and Kramkov $[7]$.

Similar problems may appear for path-dependent options such as barrier options. For instance, the delta of knock-out barrier options may explode when the maturity is small and the underlying asset is close to the barrier. This more difficult issue was recently considered by Shreve, Schmock and Wystup [11]. In this paper, the authors solve the problem of hedging a knock-out call option in a one dimensional Black-Scholes model under a constraint on the short position, i.e. the proportion of wealth invested in the risky asset is bounded from below. This result is obtained by extending the dual formulation of Cvitanic̀ and Karatzas [5] and by solving the associated stochastic control problem.

The aim of the present paper is to provide a PDE characterisation of the super-hedging price of barrier-type options. Our model is more general than the one studied in Shreve, Schmock and Wystup [11] in two aspects. First, we consider general payoffs of the form $g\left(\tau, X_{\tau}\right)$ where $\tau$ is the first exit time of a $d$-dimensional price process $X$ from a given domain $\mathcal{O}$. Secondly, our constraints on the proportions of wealth invested in the risky 
assets is described by a rather general closed convex set.

Our derivation of the associated PDE relies on the dual formulation of Cvitanic and Karatzas [5] as in Cvitanic̀, Pham and Touzi [6]. Here, the main difficulty comes from the boundary condition on $\partial \mathcal{O}$ before maturity, a problem which does not appear in the above paper. As in the vanilla option case, we have to consider as boundary condition a 'face-lifted' pay-off, but in the case of barrier options this is not sufficient. Indeed, the example considered in Shreve, Schmock and Wystup [11] shows that the boundary condition on $[0, T) \times \partial \mathcal{O}$ may not be assumed continuously by the value function, even when the payoff is 'face-lifted' (in their case $g=0$ before $T$ ). This implies that this boundary condition has to be considered in a weak sense.

In this paper, we give an appropriate sense to the boundary condition and show that the super-hedging price is a (discontinuous) viscosity solution of the corresponding Hamilton-Jacobi-Bellman equation. We also show that it can actually be further characterized as its smallest viscosity supersolution. Finally, under mild additional assumptions, we prove a comparison theorem for the associated PDE which ensures uniqueness of the solution and opens the door to the implementation of a numerical scheme. Here, the difficulty comes from the constraint on the gradient of the value function which also appears in the relaxed boundary condition. To the best of our knowledge, this is the first time that such an equation is considered.

The rest of the paper is organized as follows. The super-hedging problem and its dual formulation are presented in Section 2. In Section 3, we describe the associated PDE and state our main results. A numerical application is presented in Section 4. The remaining sections contain the proofs.

Notations: All elements $x=\left(x^{i}\right)_{i \leq d}$ of $\mathbb{R}^{d}$ are identified with column vectors with Euclydian norm $|\cdot|$ and transposed vector $x^{\prime}$. The positive orthant of $\mathbb{R}^{d}$ is denoted by $\mathbb{R}_{+}^{d}$ and the set of $d \times d$ matrices by $\mathbb{M}^{d}$. We write $\operatorname{diag}[x]$ to denote the diagonal matrix of $\mathbb{M}^{d}$ whose $i$-th diagonal element is $x^{i}$. If $y \in \mathbb{R}^{d}$, we write $x y$ for $\left(x^{i} y^{i}\right)_{i \leq d}, x^{y}$ for $\prod_{i \leq d}\left(x^{i}\right)^{y^{i}}$ and $x e^{y}$ for $\left(x^{i} e^{y^{i}}\right)_{i \leq d}$, whenever it is well defined. The trace of $M \in \mathbb{M}^{d}$ is denoted by $\operatorname{Tr}[M]$ and $|M|$ denotes its Euclydian norm when viewed as an element of $\mathbb{R}^{d^{2}}$. Given a family $\left(a^{i j}\right)_{i, j \leq d}$ of real numbers, we denote by $\left[a^{i j}\right]_{i, j}$ the matrix $A$ whose 
component $(i, j)$ is given by $a^{i j}$. The closure of a set $E \subset \mathbb{R}^{d}$ is denoted by $\bar{E}, \partial E$ stands for its boundary and $\operatorname{int}(E)$ for its interior. Given $\eta>0, B(x, \eta)$ denotes the open ball of radius $\eta$ centered on $x$.

Given a smooth function $(t, x) \in[0, T] \times \mathbb{R}^{d} \mapsto \varphi(t, x) \in \mathbb{R}$, we denote by $D \varphi$ its (partial) Jacobian matrix with respect to $x$ and by $D^{2} \varphi$ its (partial) Hessian matrix with respect to $x$. All inequalities involving random variables have to be understood in the $\mathbb{P}-$ a.s. sense.

\section{The super-hedging price under contraints and its dual formulation}

In all this paper, $T>0$ is a finite time horizon and $W=\left(W_{t}\right)_{t \leq T}$ is a $d$-dimensional Brownian motion defined on a complete probability space $(\Omega, \mathcal{F}, \mathbb{P})$. We assume that the $\mathbb{P}$-augmented filtration generated by $W, \mathbb{F}=\left(\mathcal{F}_{t}\right)_{t \leq T}$, satisfies $\mathcal{F}_{0}=\{\Omega, \emptyset\}$ and $\mathcal{F}_{T}=\mathcal{F}$.

\subsection{The barrier option hedging problem}

The financial market is composed by a non-risky asset $B$ with price process normalized to unity, i.e. $B_{t}=1$ for all $t \leq T$, and $d$ risky assets $X=\left(X^{1}, \ldots, X^{d}\right)$ whose dynamics is given by the stochastic differential equation

$$
X(t)=X_{0}+\int_{0}^{t} \operatorname{diag}[X(s)] \sigma(s, X(s)) d W_{s}, \quad t \leq T
$$

for some $X_{0} \in(0, \infty)^{d}$. Here, $\sigma:[0, T] \times \mathbb{R}_{+}^{d} \mapsto \mathbb{M}^{d}$ is assumed to satisfy

$$
\left\{\begin{array}{l}
\text { (i) } \sigma \text { is continuous, bounded and invertible with bounded inverse. } \\
\text { (ii) The map }(t, x) \in[0, T] \times \mathbb{R}_{+}^{d} \mapsto \operatorname{diag}[x] \sigma(t, x) \\
\text { is Lipschitz continuous in } x \text {, uniformly in } t
\end{array}\right.
$$

Remark 2.1 As usual there is no loss of generality in assuming that $X$ is a local martingale since, under mild assumptions on the original dynamics, we can always reduce to this case by passing to an equivalent probability measure. The normalization $B=1$ means that we consider discounted processes, i.e. we take $B$ as a numéraire. 
A financial strategy is described by a $d$-dimensional predictable process $\pi=\left(\pi^{1}, \ldots, \pi^{d}\right)$ satisfying the integrability condition

$$
\int_{0}^{T}\left|\pi_{t}\right|^{2} d t<\infty \quad \mathbb{P}-\text { a.s. }
$$

where $\pi_{t}^{i}$ is the proportion of wealth invested at time $t$ in the risky asset $X^{i}$. To an initial dotation $y \in \mathbb{R}$ and a financial strategy $\pi$, we associate the induced wealth process $Y_{y}^{\pi}$ defined as the solution on $[0, T]$ of

$$
Y(t)=y+\int_{0}^{t} Y(s) \pi_{s}^{\prime} \operatorname{diag}[X(s)]^{-1} d X(s)=y+\int_{0}^{t} Y(s) \pi_{s}^{\prime} \sigma(s, X(s)) d W_{s},
$$

where' stands for transposition.

Remark 2.2 Since in our model the financial strategies are described by the proportions of total wealth invested in each risky asset, the no-bankruptcy condition always holds provided that the initial dotation is non-negative. Indeed, it is clear from (2.4) that for $y \geq 0$, the induced wealth process satisfies $Y_{y}^{\pi}(t) \geq 0$, for all $t \in[0, T]$, a.s.

The constraints on the portfolio strategy is described by a closed convex set $K \subset \mathbb{R}^{d}$. We say that a financial strategy $\pi$ is admissible if it satisfies, in addition to the condition (2.3), the constraint

$$
\pi \in K d t \times d \mathbb{P}-\text { a.e. }
$$

and we denote by $\mathcal{K}$ the set of admissible financial strategies. All over this paper, we shall assume that

$$
0 \in K \neq \mathbb{R}^{d}
$$

The left hand-side condition just means that $0 \in \mathcal{K}$ while the inequality is natural since otherwise there would be no constraint on the portfolio.

The barrier option is described by a map $g$ defined on $[0, T] \times \mathbb{R}_{+}^{d}$ and an open domain $\mathcal{O}$ of $\mathbb{R}^{d}$ such that

$$
g \geq 0 \text { on } \overline{\mathcal{O}} \cap \mathbb{R}_{+}^{d} \quad \text { and } \quad g=0 \text { on }[0, T] \times \overline{\mathcal{O}}^{c}
$$


where $\overline{\mathcal{O}}^{c}:=(0, \infty)^{d} \backslash \overline{\mathcal{O}}$. The buyer of the option receives the payment $g(\tau, X(\tau))$ at the (stopping-) time $\tau$ defined as the first time when $X$ exists $\mathcal{O}$ if this occurs before $T$ and $T$ otherwise:

$$
\tau:=\inf \{t \in[0, T]: X(t) \notin \mathcal{O}\} \wedge T
$$

with the usual convention $\inf \emptyset=\infty$. The super-replication cost under constraint of the claim $g(\tau, X(\tau))$ is thus defined as

$$
v\left(0, X_{0}\right):=\inf \left\{y \in \mathbb{R}_{+}: Y_{y}^{\pi}(\tau) \geq g(\tau, X(\tau)) \text { for some } \pi \in \mathcal{K}\right\}
$$

Remark 2.3 The condition $g=0$ on $[0, T] \times \overline{\mathcal{O}}^{c}$ can be seen as a convention. Indeed, it is clear that $v\left(0, X_{0}\right)$ does not depend on the value of $g$ on this set when $X_{0} \in \overline{\mathcal{O}}$, while for $X_{0} \in \overline{\mathcal{O}}^{c}$ the problem has no interest.

Hereafter we present examples of barrier option which enter into our framework.

Example 2.1 Up-and-out call : Let $d=1$. The pay-off of an up-and-out call on a single asset $X^{1}$, with strike price $\kappa$ and knock-out barrier $B$ is equal to

$$
\left(X^{1}(T)-\kappa\right)^{+} \mathbf{1}_{\left\{\max _{0 \leq t \leq T} X^{1}(t)<B\right\}} .
$$

In our framework this corresponds to : $\mathcal{O}=(-\infty, B)$ and $g(t, x)=(x-\kappa)^{+} 1_{\{t=T, x<B\}}$.

Example 2.2 Down-and-out basket put option : A basket option is an option whose pay-off depends on a weighted average of a set of underlyings' values. Let $d=2$, we consider the down-and-out barrier option whose payoff is given by

$$
\left(\kappa-\frac{X^{1}(T)+X^{2}(T)}{2}\right)^{+} \mathbf{1}_{\left\{\min _{0 \leq t \leq T} X^{1}(t)+X^{2}(t)>2 B\right\}} .
$$

In our framework this correponds to $\mathcal{O}=\left\{x \in(0, \infty)^{2}, x^{1}+x^{2}>2 B\right\}$ and $g(t, x)=$ $\left(\kappa-\frac{x^{1}+x^{2}}{2}\right)^{+} 1_{\left\{t=T, x^{1}+x^{2}>2 B\right\}}$. 


\subsection{The dual formulation}

The dual formulation for hedging problems under general convex constraint was first established by Cvitanic̀ and Karatzas [5] in the diffusion case and then extended to the semi-martingale case by Föllmer and Kramkov [7], see also Karatzas and Shreve [9] and the review paper Soner and Touzi [12].

To state the dual formulation, we first need the characterization of the closed convex set $K$ in terms of its support function $\delta$. For $\rho \in R^{d}$ set

$$
\delta(\rho)=\sup _{\gamma \in K} \gamma^{\prime} \rho \geq 0
$$

where the last inequality follows from the left hand-side of (2.6), and define

$$
\tilde{K}:=\left\{\rho \in \mathbb{R}^{d}: \delta(\rho)<\infty\right\},
$$

the domain of $\delta$. Observe that the right hand-side of (2.6) implies that $\tilde{K} \neq\{0\}$. Moreover, it is a standard result of convex analysis, see e.g. [10], that $K$ can be characterized in terms of

$$
\tilde{K}_{1}:=\{\rho \in \tilde{K}:|\rho|=1\}
$$

by

$$
\gamma \in K \Leftrightarrow H(1, \gamma) \geq 0 \text { and } \gamma \in \operatorname{int}(K) \Leftrightarrow H(1, \gamma)>0
$$

where

$$
H(u, p):=\inf \left\{\delta(\rho) u-\rho^{\prime} p, \rho \in \tilde{K}_{1}\right\} \quad \text { for } \quad(u, p) \in \mathbb{R} \times \mathbb{R}^{d}
$$

Remark 2.4 Assume for a while that $0 \in \operatorname{int}(K)$. Then, there is $c_{K}>0$ such that $B\left(0, c_{K}\right) \subset K$. Thus, for all $\rho \in \tilde{K}_{1}, c_{K} \rho \in K$ and therefore

$$
\delta(\rho) \geq c_{K}>0
$$

The dual formulation is constructed as follows. Let us denote by $\tilde{\mathcal{K}}$ the set of bounded adapted processes $\vartheta$ taking values in $\tilde{K}$. To such a process, we associate the martingale $M^{\vartheta}$ defined on $[0, T]$ as the solution of

$$
M_{t}:=1+\int_{0}^{t} M_{s}\left(\sigma(s, X(s))^{-1} \vartheta_{s}\right)^{\prime} d W_{s},
$$


recall (2.2). We then define the $\mathbb{P}$-equivalent probability measure $\mathbb{Q}^{\vartheta}$ by

$$
\frac{\mathrm{d} \mathbb{Q}^{\vartheta}}{\mathrm{d} \mathbb{P}}=M_{T}^{\vartheta}
$$

It follows from Girsanov's Theorem that the process $W^{\vartheta}$ defined by

$$
W_{t}^{\vartheta}=W_{t}-\int_{0}^{t} \sigma(s, X(s))^{-1} \vartheta_{s} d s \quad t \leq T,
$$

is a Brownian motion under $\mathbb{Q}^{\vartheta}$. In the following, we shall denote by $\mathbb{E}^{\vartheta}$ the expectation operator associated to $\mathbb{Q}^{\vartheta}$.

To $\vartheta \in \tilde{\mathcal{K}}$, we finally associate the process $\mathcal{E}^{\vartheta}$ defined by

$$
\mathcal{E}_{t}^{\vartheta}:=e^{-\int_{0}^{t} \delta\left(\vartheta_{s}\right) d s} \quad t \leq T .
$$

Theorem 2.1 The following holds.

$$
v\left(0, X_{0}\right)=\sup _{\vartheta \in \tilde{\mathcal{K}}} \mathbb{E}^{\vartheta}\left[\mathcal{E}_{\tau}^{\vartheta} g(\tau, X(\tau))\right]
$$

Proof. The above result is a direct consequence of Theorem 6.2 and Remark 6.11 in [9]. For the convenience of the reader, we provide here its short proof.

1. First observe that

$$
v\left(0, X_{0}\right)=\inf \left\{y \in \mathbb{R}_{+}: Y_{y}^{\pi}(T) \geq g(\tau, X(\tau)) \quad \text { for some } \pi \in \mathcal{K}\right\}
$$

Indeed, it follows from (2.4) and condition (2.3) that, for all $y \in \mathbb{R}_{+}$and $\pi \in \mathcal{K}$, the process $Y_{y}^{\pi}$ is a non-negative local $\mathbb{P}$-martingale on $[t, T]$. Hence it is a supermartingale and, by taking conditional expectation, $Y_{y}^{\pi}(T) \geq g(\tau, X(\tau))$ implies $Y_{y}^{\pi}(\tau) \geq$ $g(\tau, X(\tau))$. From this we deduce the first inequality :

$$
v\left(0, X_{0}\right) \leq \inf \left\{y \in \mathbb{R}_{+}: Y_{y}^{\pi}(T) \geq g(\tau, X(\tau)) \quad \text { for some } \pi \in \mathcal{K}\right\} .
$$

For the converse inequality, notice that if $Y_{y}^{\pi}(\tau) \geq g(\tau, X(\tau))$, then $Y_{T}^{y, \tilde{\pi}} \geq g(\tau, X(\tau))$ where $\tilde{\pi}=\pi \mathbf{1}_{[s, \tau]}$ belongs to $\mathcal{K}$.

2. Since $g \geq 0$, see (2.7), it follows from Theorem 6.2 and Remark 6.11 in [9] that

$$
v\left(0, X_{0}\right)=\sup _{\vartheta \in \tilde{\mathcal{K}}} \mathbb{E}^{\vartheta}\left[\mathcal{E}_{T}^{\vartheta} g(\tau, X(\tau))\right] .
$$


Observe that the process $\mathcal{E}^{\vartheta}$ is positive, non-increasing in time and recall that $g \geq 0$, then the last equality leads to (2.11).

In order to derive the PDE characterization of the super-hedging price, we shall use a standard dynamic programming principle for the dual formulation of Theorem 2.1. Before to state it, we need to extend the definition of $v$ to general initial conditions $(t, x) \in[0, T] \times(0, \infty)^{d}$. For $(t, x) \in[0, T] \times(0, \infty)^{d}, y \in \mathbb{R}_{+}$and $\pi \in \mathcal{K}$, we define $\left(X_{t, x}, Y_{t, x, y}^{\pi}\right)$ as the solution of (2.1)-(2.4) on $[t, T]$ with initial condition $\left(X_{t, x}(t), Y_{t, x, y}^{\pi}(t)\right)=(x, y)$.

The value function $v$ is then defined on $[0, T] \times(0, \infty)^{d}$ by

$$
v(t, x):=\inf \left\{y \in \mathbb{R}_{+}: Y_{t, x, y}^{\pi}\left(\tau_{t, x}\right) \geq g\left(\tau_{t, x}, X_{t, x}\left(\tau_{t, x}\right)\right) \text { for some } \pi \in \mathcal{K}\right\}
$$

where

$$
\tau_{t, x}:=\inf \left\{s \in[t, T]: X_{t, x}(s) \notin \mathcal{O}\right\} \wedge T
$$

Remark 2.5 Observe that for $(t, x) \in([0, T] \times \partial \mathcal{O}) \cup(\{T\} \times \overline{\mathcal{O}})$, we have $v(t, x)=g(t, x)$ by construction.

In the sequel, we shall denote by $\mathcal{T}_{t, T}$ the set of all stopping times with values in $[t, T]$. Given $\vartheta \in \tilde{K}$ and $t<T$, we also set

$$
\mathcal{E}_{s}^{t, \vartheta}:=\mathcal{E}_{s}^{\vartheta} / \mathcal{E}_{t}^{\vartheta} \quad \text { for } s \geq t
$$

The following result is a consequence of Proposition 6.5 in [9].

Proposition 2.1 For all $(t, x) \in[0, T) \times \mathcal{O}$ and $\theta \in \mathcal{T}_{t, T}$,

$$
v(t, x)=\sup _{\vartheta \in \tilde{\mathcal{K}}} \mathbb{E}^{\vartheta}\left[\mathcal{E}_{\theta}^{t, \vartheta} v\left(\theta, X_{t, x}(\theta)\right) \mathbf{1}_{\theta<\tau_{t, x}}+\mathcal{E}_{\tau_{t, x}^{t, \vartheta}} g\left(\tau_{t, x}, X_{t, x}\left(\tau_{t, x}\right)\right) \mathbf{1}_{\theta \geq \tau_{t, x}}\right] .
$$

Proof. It follows from Proposition 6.5 in [9] that

$$
v(t, x)=\sup _{\vartheta \in \tilde{\mathcal{K}}} \mathbb{E}^{\vartheta}\left[\mathcal{E}_{\theta \wedge \tau_{t, x}}^{t, \vartheta} v\left(\theta \wedge \tau_{t, x}, X_{t, x}\left(\theta \wedge \tau_{t, x}\right)\right)\right]
$$

where by definition of $v$, see Remark 2.5, $v\left(\tau_{t, x}, X_{t, x}\left(\tau_{t, x}\right)\right)=g\left(\tau_{t, x}, X_{t, x}\left(\tau_{t, x}\right)\right)$. This provides the required result. 


\section{The PDE characterization}

Our main result consists in a PDE characterization of the value function $v$. Before to state it, we describe the PDE associated to $v$ and explain in which sense it has to be considered.

\subsection{The associated PDE}

Set $\mathcal{O}^{*}=\mathcal{O} \cap(0, \infty)^{d}$. In view of [6] and [13], it is natural to expect that the value function $v$ is a viscosity solution on

$$
D:=[0, T) \times \mathcal{O}^{*}
$$

of the partial differential equation

$$
\min \{-\mathcal{L} v, \mathcal{H} v\}=0
$$

where for a smooth function $\varphi$ on $[0, T] \times \mathbb{R}_{+}^{d}$, we set

$$
\begin{aligned}
\mathcal{H} \varphi(t, x) & =\inf \left\{\delta(\rho) \varphi(t, x)-\rho^{\prime} \operatorname{diag}[x] D \varphi(t, x), \rho \in \tilde{K}_{1}\right\} \\
\mathcal{L} \varphi(t, x) & =\frac{\partial}{\partial t} \varphi(t, x)+\frac{1}{2} \operatorname{Tr}\left[a(t, x) D^{2} \varphi(t, x)\right]
\end{aligned}
$$

with $a$ defined on $[0, T] \times \mathbb{R}_{+}^{d}$ by

$$
a(t, x):=\operatorname{diag}[x] \sigma(t, x) \sigma(t, x)^{\prime} \operatorname{diag}[x] .
$$

The first part of the equation corresponds to the usual Black-Scholes equation, while the second part is due to the portfolio constraint. Indeed, assuming that $v$ is smooth, positive, and writing formally that the hedging portfolio satisfies $Y_{y}^{\pi}(t)=v(t, X(t))$, we deduce from Itô's Lemma that $\pi_{t}$ must coincide with $\operatorname{diag}[X(t)] D v(t, X(t)) / v(t, X(t))$. Since it has to belong to $K$, the characterization of $K$ given by (2.10) implies that $H(1$, $\operatorname{diag}[X(t)] D v(t, X(t)) / v(t, X(t)))$, or equivalently $H(v(t, X(t)), \operatorname{diag}[X(t)] D v(t, X(t)))$, must be non-negative.

In order to provide a full characterization of $v$, it remains to define the boundary conditions on $\partial_{x} D^{*}:=[0, T) \times \partial \mathcal{O}^{*}$ and $\partial_{T} D^{*}:=\{T\} \times \overline{\mathcal{O}}^{*}$ where

$$
\partial \mathcal{O}^{*}:=\partial \mathcal{O} \cap(0, \infty)^{d} \quad \text { and } \quad \overline{\mathcal{O}}^{*}:=\overline{\mathcal{O}} \cap(0, \infty)^{d} .
$$


It is known from [6], see also [12] and [13], that the boundary condition on $\partial_{T} D^{*}$ has to be written

$$
v=\hat{g}
$$

where, for $x \in(0, \infty)^{d}$,

$$
\hat{g}(T, x)=\sup _{\rho \in \tilde{K}} e^{-\delta(\rho)} g\left(T, x e^{\rho}\right) .
$$

This corresponds to the 'face-lifting' procedure which was already observed by [3]. This 'face-lifting' is due to the portfolio constraint, $\hat{g}$ being the smallest function above $g$ which, in a sense, satisfies $D \hat{g} / \hat{g} \in K$.

Remark 3.1 Observe that (2.7) allows to define $\hat{g}(T, \cdot)$ on $(0, \infty)^{d}$ as

$$
\hat{g}(T, x)=\sup _{\rho \in \tilde{K}(x, \overline{\mathcal{O}})} e^{-\delta(\rho)} g\left(T, x e^{\rho}\right),
$$

with the convention $\sup \emptyset=0$ and

$$
\tilde{K}(x, E):=\left\{\rho \in \tilde{K}: x e^{\rho} \in E\right\} \quad \text { for } E \subset \overline{\mathcal{O}} .
$$

The fact that $v$ satisfy (3.1)-(3.2) in the viscosity sense can be shown by following the arguments of [6] and is not difficult.

The difficulty comes from the boundary condition on $\partial_{x} D^{*}$. In this paper, we shall show that $g$ has also to be modified on $\partial_{x} D^{*}$, i.e. replaced by $\hat{g}$ defined on $[0, T) \times(0, \infty)^{d}$ by

$$
\hat{g}(t, x)=\sup _{\rho \in \tilde{K}(x, \partial \mathcal{O})} e^{-\delta(\rho)} g\left(t, x e^{\rho}\right),
$$

with the convention $\sup \emptyset=0$. This result is expected and will be obtained under a smoothness condition on $\mathcal{O}$, see $\mathbf{H}_{\mathcal{O}}$ below.

But this is only a first step in the derivation of the appropriate boundary condition. Actually, [11] provides an example of super-hedging price for up-and-out call option for which $\hat{g}(t, x)=0$ for $t<T$ and $v\left(t^{\prime}, x^{\prime}\right)$ does not converge to 0 when $\left(t^{\prime}, x^{\prime}\right) \in D$ goes to $(t, x) \in \partial_{x} D^{*}$. This shows that the constraint on the portfolio may prevent the value function to assume the boundary condition continuously and leads to the natural formulation of a relaxed boundary condition on $\partial_{x} D^{*}$

$$
\min \{v-\hat{g}, \mathcal{H} v\}=0
$$


However, we shall see in Remark 6.1 below that the above equation has to be corrected in order to admit a viscosity supersolution and therefore have a sense. Given a smooth function $\varphi$, we therefore define

$$
\mathcal{H}_{\mathrm{d}} \varphi(t, x)=\inf \left\{\delta(\rho) \varphi(t, x)-\rho^{\prime} \operatorname{diag}[x] D \varphi(t, x), \rho \in \tilde{K}_{1}(x, \overline{\mathcal{O}})\right\},
$$

where, for $x \in E \subset \overline{\mathcal{O}}$,

$$
\tilde{K}_{1}(x, E):=\left\{\rho \in \tilde{K}_{1}: \exists \lambda_{0}>0 \text { s.t. } \lambda \rho \in \tilde{K}(x, E) \text { for all } \lambda \in\left[0, \lambda_{0}\right]\right\} .
$$

To sum up, we introduce the following operators

$$
\begin{aligned}
\mathcal{B} \varphi & :=\left\{\begin{array}{lll}
\min \{-\mathcal{L} \varphi, \mathcal{H} \varphi\} & \text { on } & D \\
\min \{\varphi-\hat{g}, \mathcal{H} \varphi\} & \text { on } & \partial_{x} D^{*} \\
\varphi-\hat{g} & \text { on } & \partial_{T} D^{*}
\end{array},\right. \\
\mathcal{B}_{\mathrm{d}} \varphi & :=\left\{\begin{array}{lll}
\mathcal{B} \varphi & \text { on } & D \cup \partial_{T} D^{*} \\
\min \left\{\varphi-\hat{g}, \mathcal{H}_{\mathrm{d}} \varphi\right\} & \text { on } & \partial_{x} D^{*}
\end{array},\right.
\end{aligned}
$$

and we say that a locally bounded function $w$ on $D$ is a discontinuous viscosity solution of

$$
\mathcal{B}_{\mathrm{d}} \varphi=0
$$

on $\bar{D}^{*}:=\bar{D} \cap\left([0, T] \times(0, \infty)^{d}\right)$ if $w_{*}$ and $w^{*}$ defined on $\bar{D}$ as

$$
w_{*}(t, x):=\liminf _{(\tilde{t}, \tilde{x}) \in D,(\tilde{t}, \tilde{x}) \rightarrow(t, x)} w(\tilde{t}, \tilde{x}) \quad \text { and } \quad w^{*}(t, x):=\limsup _{(\tilde{t}, \tilde{x}) \in D,(\tilde{t}, \tilde{x}) \rightarrow(t, x)} w(\tilde{t}, \tilde{x})
$$

are respectively viscosity super- and subsolution of $\mathcal{B}_{\mathrm{d}} \varphi=0$ and $\mathcal{B} \varphi=0$ on $\bar{D}^{*}$. More generally, we shall say that $w$ is a (discontinuous) viscosity supersolution (resp. subsolution) of $\mathcal{B} \varphi=0$ on $\bar{D}^{*}$ if $w_{*}$ is a supersolution of $\mathcal{B}_{\mathrm{d}} \varphi=0$ (resp. subsolution of $\mathcal{B} \varphi=0)$ on $\bar{D}^{*}$.

Remark 3.2 Assume that the conditions of Theorem 3.1 below hold. Let us write $\mathcal{B} \varphi(t, x)$ as $\mathcal{B}\left(t, x, \varphi(t, x), \frac{\partial}{\partial t} \varphi(t, x), D \varphi(t, x), D^{2} \varphi(t, x)\right)$ and $\mathcal{B}_{\mathrm{d}} \varphi(t, x)$ similarly. Then, one easily checks that the upper-semicontinuous envelope of $\mathcal{B}_{\mathrm{d}}$ as a map on $\bar{D}^{*} \times \mathbb{R} \times$ $\mathbb{R} \times \mathbb{R}^{d} \times \mathbb{M}^{d}$ is given by

$$
\begin{aligned}
\left.\left(\mathcal{B}_{\mathrm{d}}\right)_{+}\left(t, x, \varphi(t, x), \frac{\partial}{\partial t} \varphi(t, x), D \varphi(t, x), D^{2} \varphi(t, x)\right)\right)= & \max \left\{\mathcal{B}_{\mathrm{d}} \varphi(t, x),\right. \\
& \min \{-\mathcal{L} \varphi(t, x), \mathcal{H} \varphi(t, x)\}\},
\end{aligned}
$$


and that its lower-semicontinuous envelope is

$$
\left.\left(\mathcal{B}_{\mathrm{d}}\right)_{-}\left(t, x, \varphi(t, x), \frac{\partial}{\partial t} \varphi(t, x), D \varphi(t, x), D^{2} \varphi(t, x)\right)\right)=\min \{\mathcal{B} \varphi(t, x),-\mathcal{L} \varphi(t, x)\} .
$$

From the arguments of the proof of Proposition 6.3 and Proposition 6.6 below, we deduce that $\left(\mathcal{B}_{\mathrm{d}}\right)_{+} \varphi=0$ (resp. $\left.\left(\mathcal{B}_{\mathrm{d}}\right)_{-} \varphi=0\right)$ has the same supersolutions as $\mathcal{B}_{\mathrm{d}} \varphi=0$ (resp. subsolutions that $\mathcal{B} \varphi=0$ ) on $D \cup \partial_{x} D^{*}$, for the terminal condition $\varphi=\hat{g}$ at $T$. In other words, $\mathcal{B}_{\mathrm{d}}$ can be viewed as being upper-semicontinuous with lowersemicontinuous envelope given by $\mathcal{B}$. This justifies the above definition of a viscosity solution of $\mathcal{B}_{\mathrm{d}} \varphi=0$, and shows that it is in accordance with Definition 7.4 in [4]. This remark will be used in the example section to prove the convergence of the discretization scheme we shall consider for a particular example.

\subsection{Main results}

In order to establish that $v$ is a discontinuous viscosity solution of (3.8), we shall appeal to the following additional assumptions.

Our first condition concerns the convex set $K$ describing the portfolio constraints. It is stated in terms of $\tilde{K}(x, \mathcal{O})$, recall (3.4).

$$
\begin{aligned}
& \mathbf{H}_{\tilde{K}}: \quad \text { (i) } \text { For all } x \in \mathcal{O}, \rho \in \tilde{K}(x, \mathcal{O}) \text { implies } \lambda \rho \in \tilde{K}(x, \mathcal{O}) \text { for all } \lambda \in[0,1) . \\
& \text { (ii) } \text { For all } x \in \mathcal{O} \text {, the closure of } \tilde{K}(x, \mathcal{O}) \text { is equal to } \tilde{K}(x, \overline{\mathcal{O}}) . \\
& \text { (iii) } \text { If }\left(x_{n}\right)_{n} \text { is a sequence in } \mathcal{O} \text { such that } x_{n} \rightarrow x \in \partial \mathcal{O} \text { and } \rho \in \tilde{K}(x, \overline{\mathcal{O}}) \\
& \text { then there exists a sequence } \rho_{n} \rightarrow \rho \text { such that, up to a subsequence, } \\
& \\
& \rho_{n} \in \tilde{K}\left(x_{n}, \overline{\mathcal{O}}\right) \forall n \geq 1 .
\end{aligned}
$$

Remark 3.3 The conditions $(i)$ and (ii) of $\mathbf{H}_{\tilde{K}}$ are automatically satisfied whenever the set $\ln (\mathcal{O})=\left\{\left(\ln \left(x^{i}\right)\right)_{i \leq d}, x \in \mathcal{O}\right\}$ is convex. Indeed, we easily check that in this case, for all $x \in \mathcal{O}, \tilde{K}(x, \mathcal{O})$ is convex, and since $0 \in \tilde{K}(x, \mathcal{O})$, this provides $(i)$. The convexity of $\ln (\mathcal{O})$ also implies that if $\rho \in \tilde{K}(x, \mathcal{O})$ and $\bar{\rho} \in \tilde{K}(x, \overline{\mathcal{O}})$, then $\lambda \rho+(1-\lambda) \bar{\rho} \in \tilde{K}(x, \mathcal{O})$ for all $\lambda \in(0,1)$. Since $0 \in \tilde{K}$, this shows that for all $x \in \mathcal{O}$ the closure of $\tilde{K}(x, \mathcal{O})$ contains $\tilde{K}(x, \overline{\mathcal{O}})$, while the converse inclusion is obvious. 
We shall also impose some regularity assumptions on $g$ :

$\mathbf{H}_{g}: \quad$ (i) $\quad g$ is lower semi-continuous on $[0, T] \times \partial \mathcal{O}^{*}$ and on $\{T\} \times \overline{\mathcal{O}}^{*}$.

(ii) $\exists C_{g}>0$ and $\bar{\gamma} \in K \cap \mathbb{R}_{+}^{d}$ s.t. $|g(\cdot, x)| \leq C_{g}\left(1+x^{\bar{\gamma}}\right) \forall x \in \overline{\mathcal{O}}^{*}$,

(iii) $\hat{g}$ is upper semi-continuous on $[0, T] \times(0, \infty)^{d}$ and has linear growth.

Under $\mathbf{H}_{\tilde{K}}$ and (i)-(ii) of $\mathbf{H}_{g}$, one can already derive the following qualitative properties of $v$.

Proposition 3.1 Assume that $\mathbf{H}_{\tilde{K}}$ and (i)-(ii) of $\mathbf{H}_{g}$ hold. Then, for all $(t, x) \in D$, we have

$$
v(t, x) \geq 0
$$

and there is a constant $C>0$, independent of $(t, x)$, such that

$$
|v(t, x)| \leq C\left(1+x^{\bar{\gamma}}\right)
$$

Moreover, for all $(t, x) \in \bar{D}$,

$$
v_{*}(t, x)=\sup _{\rho \in \tilde{K}(x, \overline{\mathcal{O}})} e^{-\delta(\rho)} v_{*}\left(t, x e^{\rho}\right) .
$$

The proof will be provided in Section 5 .

In order to derive the appropriate boundary condition on $\partial_{x} D^{*}$, we shall also need some regularity on the domain $\mathcal{O}$.

$\mathbf{H}_{\mathcal{O}}: \quad$ There exists a map $d:(0, \infty)^{d} \mapsto \mathbb{R}$ such that

(i) $\left\{x \in(0, \infty)^{d}: d(x)>0\right\}=\mathcal{O}^{*}$.

(ii) $\left\{x \in(0, \infty)^{d}: d(x)=0\right\}=\partial \mathcal{O}^{*}$.

(iii) $\forall x \in \partial \mathcal{O}^{*},, \exists r>0$ s.t. $d \in C^{2}(B(x, r))$.

This essentially amongs to say that $\mathcal{O}$ is $C^{2}$, see [8].

Using $\mathbf{H}_{\tilde{K}}, \mathbf{H}_{g}$ and $\mathbf{H}_{\mathcal{O}}$, we can already characterize $v$ not only as a discontinuous solution of (3.8) but also as its smallest supersolution. 
Theorem 3.1 Assume that $\mathbf{H}_{\tilde{K}}, \mathbf{H}_{g}$ and $\mathbf{H}_{\mathcal{O}}$ hold. Then,

(i) $\quad v$ is a discontinuous viscosity solution of (3.8),

(ii) $v$ is lower-continuous on $D$,

(iii) $v_{*}$ is the smallest supersolution of (3.8) in the class of locally bounded functions satisfying (3.10).

Finally, under the additional assumptions

$$
\begin{gathered}
\mathbf{H}^{\prime}: \text { (i) Either } \overline{\mathcal{O}} \text { is bounded or } \exists \varrho>1 \text { s.t. } \varrho \bar{\gamma} \in K \cap(0, \infty)^{d}, \\
\text { (ii) } \operatorname{int}(K) \neq \emptyset \text { and either } 0 \in \operatorname{int}(K) \text { or } \overline{\mathcal{O}} \cap \partial \mathbb{R}_{+}^{d}=\emptyset, \\
\text { (iii) } \forall x \in \partial \mathcal{O}^{*} \exists \rho \in \tilde{K}_{1} \text { s.t. } D d(x)^{\prime} \operatorname{diag}[x] \rho>0,
\end{gathered}
$$

we will be able in Section 7 to provide a comparison theorem for (3.8). It will imply our last result which characterizes $v$ as the unique solution of (3.8) in a suitable class of functions.

Theorem 3.2 Let the conditions of Theorem 3.1 hold and assume further that $\mathbf{H}^{\prime}$ is satisfied. Then,

(i) $v_{*}=v^{*}$ on $\bar{D}^{*}$,

(ii) $v$ is continuous on $D$,

(iii) $v$ is the unique discontinuous viscosity solution of (3.8) in the class of locally bounded function satisfying (3.10).

Remark 3.4 Recall the examples of barrier options of Section 2.

1. If we hedge the up-and-out call of the Example 2.1 with shortsales constaints, i.e. $K=[-\alpha,+\infty)$, with $\alpha>0$, then it is easy to verify that all of the conditions $\mathbf{H}_{\tilde{K}}, \mathbf{H}_{g}$, $\mathbf{H}_{\mathcal{O}}$ and $\mathbf{H}^{\prime}$ hold true.

2. These conditions are also satisfied when we hedge the down-and-out basket put of the Example 2.2 with bounded portfolio, i.e $K=\prod_{i=1}^{2}\left[-\underline{\alpha^{i}}, \bar{\alpha}^{i}\right], \underline{\alpha^{i}}, \bar{\alpha}^{i}>0$ for $i=1,2$.

Remark 3.5 To conclude this section, let us comment the assumption $\mathbf{H}^{\prime}$. As already mentioned, Theorem 3.2 is based on a comparison result for (3.8) stated in Theorem 7.1 below. A first difficulty in proving this theorem comes from the growth condition (3.10) which is non-standard. In the case where $\overline{\mathcal{O}}$ is not bounded, the second assumption in (i) is used to construct a suitable penalty function which allows us to reduce to a 
bounded domain. The second difficulty comes from the term $\mathcal{H} \varphi$ appearing in $\mathcal{B} \varphi$. It is handled by using the first assertion of (ii) under which we can construct a strict super-solution of $\mathcal{H} \varphi=0$. A third difficulty is due to the fact that the equation in written only on $\overline{\mathcal{O}} \cap(0, \infty)^{d}$. In the case where $\overline{\mathcal{O}} \cap \partial \mathbb{R}_{+}^{d} \neq \emptyset$, we need to introduce an other penalty function which permits to reduce the analysis to $(0, \infty)^{d}$. We then appeal to the second assertion of (ii). Finally, a major difficulty comes from the boundary condition on $\partial_{x} D^{*}$ which is written in a weak sense. It is treated by using the condition (iii) which allows to "avoid" this boundary. We refer to step 4. of the proof of Theorem 7.1 below for a more detailed discussion of these assumptions (i) and (ii).

\section{A numerical application}

In this section, we study a numerical scheme for the resolution of $\mathcal{B}_{\mathrm{d}} \varphi=0$ in the simple example considered in [11] : superhedging of a knock-out call with a short-sale constraint. The general case will be discussed in the companion paper [2].

The model corresponds to our general framework with $d=1, \sigma(t, x)=\sigma>0$, a fixed constant, $\mathcal{O}=(-\infty, B), K=[-\alpha, \infty)$ and $g(t, x)=[x-\kappa]^{+} \mathbf{1}_{\{t=T, x<B\}}$, with $\alpha>0$, $B>\kappa>0$. In this case $\tilde{K}=(-\infty, 0]$, the function $\hat{g}(t, x)$ is equal to

$$
\hat{g}(t, x)=e^{-\alpha \theta(x)}\left[x e^{-\theta(x)}-K\right]^{+} \mathbf{1}_{t=T} \quad \text { with } \quad \theta(x)=[-\ln (B / x)]^{+},
$$

and all the assumptions of Theorem 3.2 are satisfied.

In order to solve numerically the equation $\mathcal{B}_{\mathrm{d}} \varphi=0$, we propose the following discretization. We fix a regular grid $\pi_{h}=\left\{t_{i}:=\left(i r_{h}\right) \wedge T, 0 \leq i \leq I_{h}\right\}$ of $[0, T]$ and $\Xi_{h}:=\left\{x_{i}:=(i h) \wedge B, 0 \leq i \leq N_{h}\right\}$ of $[0, B]$. Here, $h>0$ is a fixed parameter, $N_{h}:=\inf \{k \in \mathbb{N}: k \geq B / h\}$ and $I_{h}:=\inf \left\{k \in \mathbb{N}: k \geq T / r_{h}\right\}$ with $r_{h}=h^{2}$. The approximation $v^{h}$ of $v$ is defined as follows.

1. For $i=I_{h}$, we use the boundary condition at $t=T$ to set $: v^{h}\left(t_{I_{h}}, x_{j}\right)=\hat{g}\left(t_{I_{h}}, x_{j}\right)$, $j=0, \ldots, N_{h}$.

2. For $i=I_{h}-1, \ldots, 0$, we use the following procedure :

2.a. We initialize $: v^{h}\left(t_{i}, 0\right)=0$.

2.b. Then, we solve on $j=1, \ldots, N_{h}$ the system

$$
v^{h}\left(t_{i}, x_{j}\right)=\left\{\begin{array}{lr}
\max \left\{A^{h}\left(v^{h}, i, j\right) ; B^{h}\left(v^{h}, i, j\right)\right\} & \text { if } j \neq N_{h} \\
\max \left\{0 ; B^{h}\left(v^{h}, i, j\right)\right\} & \text { otherwise }
\end{array}\right.
$$


with

$$
\begin{aligned}
A^{h}\left(v^{h}, i, j\right) & :=\frac{\left(r_{h}\right)^{-1} v^{h}\left(t_{i+1}, x_{j}\right)+\left(2 h^{2}\right)^{-1} \sigma^{2} x_{j}^{2}\left(v^{h}\left(t_{i}, x_{j+1}\right)+v^{h}\left(t_{i}, x_{j-1}\right)\right)}{\left(r_{h}\right)^{-1}+\left(h^{2}\right)^{-1} \sigma^{2} x_{j}^{2}} \\
B^{h}\left(v^{h}, i, j\right) & :=\frac{x_{j} h^{-1} v^{h}\left(t_{i}, x_{j-1}\right)}{\alpha+x_{j} h^{-1}},
\end{aligned}
$$

The initialization of step 2.a. is justified by the continuity of $v$ at 0 which is easily checked in this simple model by using the dual formulation of Theorem 2.1. The system given in 2.b. follows from the approximation of $\mathcal{H}=\mathcal{H}_{d}$ and $\mathcal{L}$ by

$$
\begin{aligned}
\mathcal{H}^{h}\left(t_{i}, x_{j}, v^{h}\left(t_{i}, x_{j}\right), v^{h}\right) & =\alpha v^{h}\left(t_{i}, x_{j}\right)+x_{j} \frac{v^{h}\left(t_{i}, x_{j}\right)-v^{h}\left(t_{i}, x_{j-1}\right)}{h}, \\
\mathcal{L}^{h}\left(t_{i}, x_{j}, v^{h}\left(t_{i}, x_{j}\right), v^{h}\right) & =\frac{v^{h}\left(t_{i+1}, x_{j}\right)-v^{h}\left(t_{i}, x_{j}\right)}{r_{h}} \\
& +\frac{1}{2} \sigma^{2} x_{j}^{2} \frac{v^{h}\left(t_{i}, x_{j+1}\right)+v^{h}\left(t_{i}, x_{j-1}\right)-2 v^{h}\left(t_{i}, x_{j}\right)}{h^{2}} .
\end{aligned}
$$

Observing that $v^{h}$ is non-negative and uniformly bounded from above by $B$, the convergence of the scheme easily follows from Remark 3.2, Theorem 3.2, Remark 7.1 below and [1].

Figure 1:
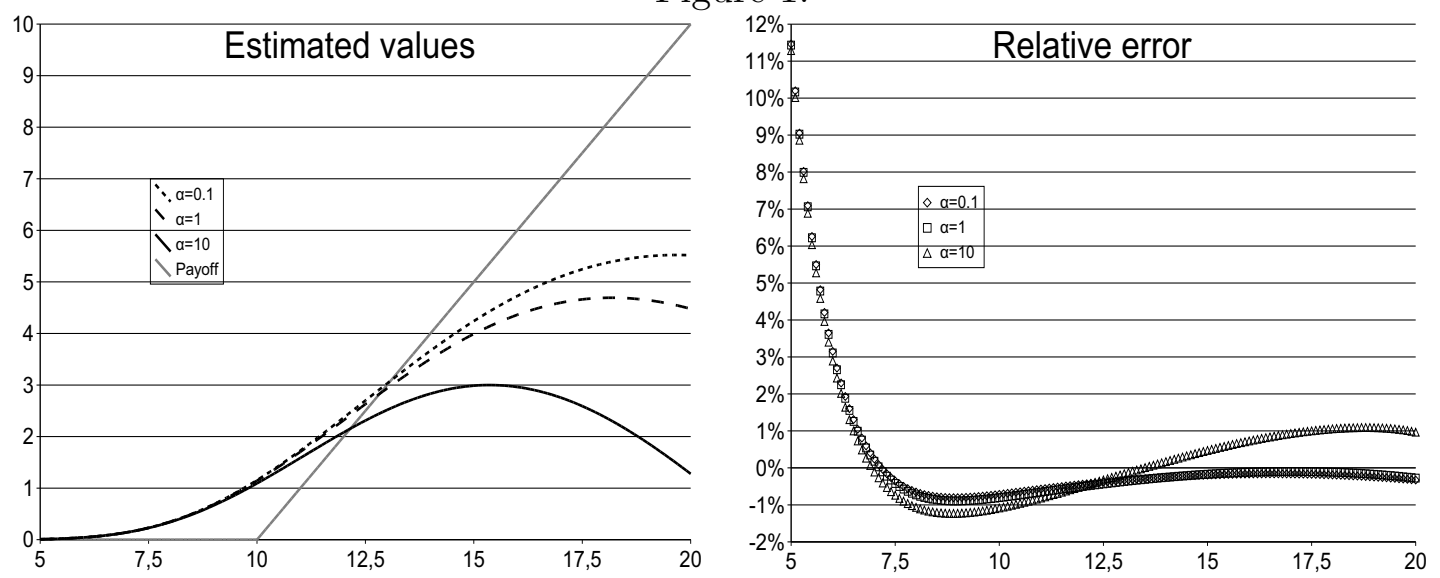

In Figure 1, we plot the estimation of $v$ obtained with this scheme for $\sigma=0.3, \kappa=10$, $B=20, T=1$ and for $\alpha \in\{0.1,1,10\}$. The relative error is computed by using 
the closed form solution obtained in [11]. We took $N_{h}=200$. We observe that the estimation is very sharp with a relative error less than $1 \%$ in absolute value, except for small values of $X_{0}$ for which $v$ is almost equal to 0 .

\section{Growth and monotonicity properties}

In this section, we provide the proof of Proposition 3.1.

Proof of (3.9)-(3.10). The lower bound of (3.9) is an immediate consequence of the assumption $g \geq 0$ and the dual formulation (2.11). We now prove (3.10). Let $\pi \in \mathcal{K}$ be defined by $\pi_{t}=\bar{\gamma}$ for all $t \leq T$. Since $\sigma$ is bounded, see (2.2), one easily checks from the dynamics of the processes $X_{t, x}$ and $Y_{t, x, 1}^{\pi}$ that

$$
1+\prod_{i=1}^{d}\left(X_{t, x}^{i}(u)\right)^{\bar{\gamma}^{i}} \leq C\left(1+\prod_{i=1}^{d}\left(x^{i}\right)^{\bar{\gamma}^{i}}\right) Y_{t, x, 1}^{\pi}(u) \quad \text { for all } u \in[0, T], \mathbb{P}-\text { a.s. },
$$

where $C>0$ depends only on $|\bar{\gamma}|$ and the bound on $|\sigma|$. Then, after possibly changing the value of the constant $C, \mathbf{H}_{g^{-}}$(ii) implies

$$
g\left(u, X_{t, x}(u)\right) \leq C\left(1+x^{\bar{\gamma}}\right) Y_{t, x, 1}^{\pi}(u) \quad \text { for all } u \in[0, T], \mathbb{P}-\text { a.s. },
$$

and since $y Y_{t, x, 1}^{\pi}=Y_{t, x, y}^{\pi}$ for $y>0$, we deduce from the last inequality that $v(t, x) \leq$ $C\left(1+x^{\bar{\gamma}}\right)$.

Proof of $(3.11)$. Since $0 \in \tilde{K}(x, \overline{\mathcal{O}})$, we only have to show that

$$
v_{*}(t, x) \geq \sup _{\rho \in \tilde{K}(x, \overline{\mathcal{O}})} e^{-\delta(\rho)} v_{*}\left(t, x e^{\rho}\right) .
$$

1. We first consider the case where $(t, x) \in D$. Since by lower-semicontinuity of $v_{*}$ and (ii) of $\mathbf{H}_{\tilde{K}}$

$$
\sup _{\rho \in \tilde{K}(x, \mathcal{O})} e^{-\delta(\rho)} v_{*}\left(t, x e^{\rho}\right)=\sup _{\rho \in \tilde{K}(x, \overline{\mathcal{O}})} e^{-\delta(\rho)} v_{*}\left(t, x e^{\rho}\right)
$$

it suffices to show that

$$
v_{*}(t, x) \geq \sup _{\rho \in \tilde{K}(x, \mathcal{O})} e^{-\delta(\rho)} v_{*}\left(t, x e^{\rho}\right)
$$


Fix $\rho \in \tilde{K}(x, \mathcal{O})$ and consider the sequence of processes $\vartheta^{n}$ in $\tilde{\mathcal{K}}$ defined on $[t, T]$ by $\vartheta^{n}:=n \rho \mathbf{1}_{\left[t, t_{n}\right]}$ with $t_{n}:=t+n^{-1}$ for $n$ large enough so that $t_{n}<T$. By Proposition 2.1 $v(t, x) \geq \mathbb{E}^{\vartheta^{n}}\left[e^{-n \delta(\rho)\left(n^{-1} \wedge\left(\tau_{t, x}-t\right)\right)}\left(v\left(t_{n}, X_{t, x}\left(t_{n}\right)\right) \mathbf{1}_{t_{n}<\tau_{t, x}}+g\left(\tau_{t, x}, X_{t, x}\left(\tau_{t, x}\right)\right) \mathbf{1}_{t_{n} \geq \tau_{t, x}}\right)\right]$.

Let $X^{n}$ be the solution on $[t, T]$ of

$$
X^{n}(s)=x+\int_{t}^{s} \operatorname{diag}\left[X^{n}(r)\right] \vartheta_{r}^{n} d r+\int_{t}^{s} \operatorname{diag}\left[X^{n}(r)\right] \sigma\left(r, X^{n}(r)\right) d W_{r}
$$

so that $X^{n}(s)=\beta_{s}^{n} H_{s}^{n}$ with

$$
\left(H_{s}^{n}\right)^{i}:=\mathcal{E}\left(\sum_{j=1}^{d} \int_{t}^{s} \sigma^{i j}\left(r, X^{n}(r)\right) d W_{r}^{j}\right) \quad \text { and } \quad \beta_{s}^{n}:=x e^{\int_{t}^{s} \vartheta_{r}^{n} d r},
$$

where $\mathcal{E}$ denotes the Doleans-Dade exponential. By Girsanov's theorem, (5.2) can be rewritten as

$$
v(t, x) \geq \mathbb{E}\left[e^{-n \delta(\rho)\left(n^{-1} \wedge\left(\tau_{n}-t\right)\right)}\left(v\left(t_{n}, X^{n}\left(t_{n}\right)\right) \mathbf{1}_{t_{n}<\tau_{n}}+g\left(\tau_{n}, X^{n}\left(\tau_{n}\right)\right) \mathbf{1}_{t_{n} \geq \tau_{n}}\right)\right]
$$

where

$$
\tau_{n}:=\inf \left\{s \in[t, T]: X^{n}(s) \notin \mathcal{O}\right\} \wedge T .
$$

Since $\sigma$ is bounded, see $(2.2), H_{\tau_{n} \wedge t_{n}}^{n} \rightarrow(1, \ldots, 1) \mathbb{P}-$ a.s., after possibly passing to a subsequence. Also observe that

$$
\beta_{\tau_{n} \wedge t_{n}}^{n}=x e^{\rho\left[\left(n\left(\tau_{n}-t\right)\right) \wedge 1\right]} .
$$

By $\mathbf{H}_{\tilde{K}}$ and the assumption $\rho \in \tilde{K}(x, \mathcal{O})$, it follows that, $\mathbb{P}-$ a.s., $X_{\tau_{n} \wedge t_{n}}^{n} \in \mathcal{O}$ and therefore $t_{n}<\tau_{n}$ for large values of $n$. In particular,

$$
\left(X^{n}\left(t_{n}\right), \mathbf{1}_{t_{n}<\tau^{n}}\right) \longrightarrow\left(x e^{\rho}, 1\right) \mathbb{P}-\text { a.s. }
$$

Thus, passing to the limit in (5.3) and applying Fatou's Lemma shows the required result, recall (3.9).

2. We now consider the case where $(t, x) \in \partial D$. Let $\left(t_{n}, x_{n}\right)_{n}$ be a sequence in $D$ that converges to $(t, x)$ such that $v\left(t_{n}, x_{n}\right) \rightarrow v_{*}(t, x)$. Fix $\rho \in \tilde{K}(x, \overline{\mathcal{O}})$. By $\mathbf{H}_{\tilde{K}}$, there is a sequence $\left(\rho_{n}\right)_{n}$ with values in $\tilde{K}\left(x_{n}, \overline{\mathcal{O}}\right)$ such that $\rho_{n} \rightarrow \rho$. Using 1., we deduce that $v\left(t_{n}, x_{n}\right) \geq e^{-\delta\left(\rho_{n}\right)} v_{*}\left(t_{n}, x_{n} e^{\rho_{n}}\right)$. Passing to the limit shows that $v_{*}(t, x) \geq e^{-\delta(\rho)} v_{*}\left(t, x e^{\rho}\right)$ by lower-semicontinuity of $v_{*}$. 
Remark 5.1 Fix $(t, x) \in \bar{D}^{*}$ and assume that $\left(\lambda_{0}, \rho_{0}\right) \in \mathbb{R}_{+} \times \tilde{K}$ are such that $x e^{\lambda_{0} \rho_{0}} \in$ $\overline{\mathcal{O}}$. By (i) $\mathbf{H}_{\tilde{K}}$, the map $\lambda \in\left[0, \lambda_{0}\right] \mapsto e^{-\lambda \delta\left(\rho_{0}\right)} v_{*}\left(t, x e^{\lambda \rho_{0}}\right)$ is well defined and it follows from (3.11) that it is non-increasing.

\section{The viscosity solution property}

In this section, we provide the proof of Theorem 3.1. We start with the supersolution and subsolution properties. Then, we use an approximation argument combined with a comparison theorem to prove that $v_{*}$ is the smallest supersolution of (3.8).

\subsection{Supersolution property}

In this section, we show that $v_{*}$ is a supersolution of $(3.8)$ on $\bar{D}^{*}$. This is a consequence of Proposition 6.1, 6.2, 6.3 and 6.4 below.

Proposition 6.1 Assume that $\mathbf{H}_{\tilde{K}^{-}}-\mathbf{H}_{g}$ hold. Let $\left(t_{0}, x_{0}\right) \in \bar{D}^{*}$ and $\varphi \in C^{2}\left(\bar{D}^{*}\right)$ be such that $\left(t_{0}, x_{0}\right)$ is a local minimum on $\bar{D}^{*}$ of $v_{*}-\varphi$ satisfying $\left(v_{*}-\varphi\right)\left(t_{0}, x_{0}\right)=0$. Then,

$$
\mathcal{H}_{\mathrm{d}} \varphi\left(t_{0}, x_{0}\right) \geq 0
$$

Proof. By (3.11), for all $\rho \in \tilde{K}_{1}(x, \overline{\mathcal{O}})$ and $\lambda>0$ small enough, we must have

$$
\varphi\left(t_{0}, x_{0}\right)=v_{*}\left(t_{0}, x_{0}\right) \geq e^{-\lambda \delta(\rho)} v_{*}\left(t_{0}, x_{0} e^{\lambda \rho}\right) \geq e^{-\lambda \delta(\rho)} \varphi\left(t_{0}, x_{0} e^{\lambda \rho}\right) .
$$

Thus, dividing by $\lambda$ and sending $\lambda$ to 0 leads to the required result.

Remark 6.1 Assume that $\mathbf{H}_{\mathcal{O}}$ holds and that for all $\left(t_{0}, x_{0}\right) \in \bar{D}^{*}$ and $\varphi$ as in Proposition 6.1 , we have

$$
\mathcal{H} \varphi\left(t_{0}, x_{0}\right) \geq 0
$$

Let $\left(t_{0}, x_{0}\right)$ and $\varphi$ be as in Proposition 6.1 with $x_{0} \in \partial \mathcal{O}^{*}$. Recall from $\mathbf{H}_{\mathcal{O}}$ the definition of the function $d$ and observe that $\left(t_{0}, x_{0}\right)$ is also a local minimum of $\left(v_{*}-\varphi\right)(t, x)+$ $\varepsilon^{-1} d(x)$ on $\bar{D}^{*}$ for all $\varepsilon>0$. Thus, if the above assertion is true, $\varphi-\varepsilon^{-1} d$ must satisfy

$$
\delta(\rho) v_{*}\left(t_{0}, x_{0}\right)-\rho^{\prime} \operatorname{diag}\left[x_{0}\right]\left(D \varphi\left(t_{0}, x_{0}\right)-\varepsilon^{-1} D d\left(x_{0}\right)\right) \geq 0 \quad \text { for all } \rho \in \tilde{K}_{1} .
$$


Now observe that for $\rho \in \tilde{K}_{1} \backslash \tilde{K}_{1}\left(x_{0}, \overline{\mathcal{O}}\right)$, there is a sequence of positive parameters $\lambda_{n} \rightarrow 0$ such that $d\left(x_{0} e^{\lambda_{n} \rho}\right)<0=d\left(x_{0}\right)$ for all $n$, recall (3.7). This implies that $\rho^{\prime} \operatorname{diag}\left[x_{0}\right] D d\left(x_{0}\right)<0$. Hence, sending $\varepsilon \rightarrow 0$ in the above inequality leads to a contradiction if $\tilde{K}_{1} \backslash \tilde{K}_{1}\left(x_{0}, \overline{\mathcal{O}}\right) \neq \emptyset$.

Proposition 6.2 Let $\left(t_{0}, x_{0}\right) \in D$ and $\varphi \in C^{2}\left(\bar{D}^{*}\right)$ be such that $\left(t_{0}, x_{0}\right)$ is a local minimum on $\bar{D}^{*}$ of $v_{*}-\varphi$ satisfying $\left(v_{*}-\varphi\right)\left(t_{0}, x_{0}\right)=0$. Then,

$$
-\mathcal{L} \varphi\left(t_{0}, x_{0}\right) \geq 0
$$

Proof. The proof is standard. Let $V$ be a bounded open neighborhood of $\left(t_{0}, x_{0}\right)$ such that $\left(t_{0}, x_{0}\right)$ is a minimum on $\bar{V} \cap \bar{D}^{*}$ of $v_{*}-\varphi$ and let $\left(t_{n}, x_{n}\right)_{n}$ be a sequence in $V \cap D$ such that $\left(t_{n}, x_{n}\right) \rightarrow\left(t_{0}, x_{0}\right)$ and $v\left(t_{n}, x_{n}\right) \rightarrow v_{*}\left(t_{0}, x_{0}\right)$. For ease of notations we write $\left(\tau_{n}, X^{n}\right)=\left(\tau_{t_{n}, x_{n}}, X_{t_{n}, x_{n}}\right)$. Given a sequence $\left(\eta_{n}\right)_{n}$ of positive numbers such that $t_{n}+\eta_{n}<T$ for all $n$, we set

$$
\theta_{n}:=\inf \left\{s \in\left[t_{n}, T\right]:\left(s, X^{n}(s)\right) \notin V \cap D\right\} \wedge\left(t_{n}+\eta_{n}\right) .
$$

Since $0 \in \tilde{K},(2.13)$, the assumption $g \geq 0$, see (2.7), and the inequality $v_{*} \geq \varphi$ on $V$ imply that

$$
v\left(t_{n}, x_{n}\right) \geq \mathbb{E}\left[\varphi\left(\theta_{n}, X_{\theta_{n}}^{n}\right) \mathbf{1}_{\theta_{n}<\tau_{n}}\right]
$$

Set $\epsilon_{n}:=v\left(t_{n}, x_{n}\right)-\varphi\left(t_{n}, x_{n}\right)$ and observe that $\epsilon_{n}$ converges to 0 as $n$ goes to infinity. Moreover, it follows from Itô's Lemma that

$$
\epsilon_{n} \geq \mathbb{E}\left[\int_{t_{n}}^{\theta_{n}} \mathcal{L} \varphi\left(s, X^{n}(s)\right) d s \mathbf{1}_{\theta_{n}<\tau_{n}}\right]
$$

Using standard estimates, we then observe that

$$
\liminf _{n \rightarrow \infty} \mathbb{E}\left[\eta_{n}^{-1} \int_{t_{n}}^{\theta_{n}} \mathcal{L} \varphi\left(s, X^{n}(s)\right) d s \mathbf{1}_{\theta_{n}<\tau_{n}}\right] \geq \mathcal{L} \varphi\left(t_{0}, x_{0}\right)
$$

whenever $\eta_{n} \rightarrow 0$. Thus, choosing $\left(\eta_{n}\right)_{n}$ such that $\epsilon_{n} / \eta_{n} \rightarrow 0$ and using (6.2) leads to the required result.

Proposition 6.3 Assume that $\mathbf{H}_{\tilde{K}}-\mathbf{H}_{g}$ holds. Then, $v_{*} \geq \hat{g}$ on $\partial_{x} D^{*}$. 
Proof. 1. We first prove that for all $\left(t_{0}, x_{0}\right) \in \partial_{x} D^{*}$ and $\varphi \in C^{2}\left(\bar{D}^{*}\right)$ such that

$$
0=\left(v_{*}-\varphi\right)\left(t_{0}, x_{0}\right)=\min _{\bar{D}^{*}}(\text { strict })\left(v_{*}-\varphi\right)
$$

we have

$$
\max \left\{v_{*}\left(t_{0}, x_{0}\right)-g\left(t_{0}, x_{0}\right) ;-\mathcal{L} \varphi\left(t_{0}, x_{0}\right)\right\} \geq 0
$$

Assume to the contrary that

$$
\max \left\{\varphi\left(t_{0}, x_{0}\right)-g\left(t_{0}, x_{0}\right) ;-\mathcal{L} \varphi\left(t_{0}, x_{0}\right)\right\} \leq-2 \varepsilon
$$

for some $\varepsilon>0$. Let $\left(t_{n}, x_{n}\right)_{n}$ be a sequence in $D$ converging to $\left(t_{0}, x_{0}\right)$ such that

$$
v\left(t_{n}, x_{n}\right) \rightarrow v_{*}\left(t_{0}, x_{0}\right)
$$

By (2.2) and $\mathbf{H}_{g}$, there is an open ball $B$ centered on $\left(t_{0}, x_{0}\right)$ such that

$$
-\mathcal{L} \varphi \leq 0 \text { on } B \cap \bar{D}^{*} \quad \text { and } \quad \varphi-g \leq-\varepsilon \text { on } B \cap \partial_{x} D^{*}
$$

Obviously, we can assume that $\left(t_{n}, x_{n}\right) \in B$. Set $\left(\tau_{n}, X^{n}\right)=\left(\tau_{t_{n}, x_{n}} X_{t_{n}, x_{n}}\right)$ and let $\theta_{n}$ be the first exit time of $\left(X^{n}(s)\right)_{s \geq t_{n}}$ from $B$. Observing that $\xi:=\min _{\partial B \cap \bar{D}}\left(v_{*}-\varphi\right)>0$, using Itô's Lemma and (6.5) one obtains

$$
\begin{aligned}
\varphi\left(t_{n}, x_{n}\right) & \leq \mathbb{E}\left[\varphi\left(\tau_{n} \wedge \theta_{n}, X^{n}\left(\tau^{n} \wedge \theta^{n}\right)\right)\right] \\
& \left.\leq-(\varepsilon \wedge \xi)+\mathbb{E}\left[g\left(\tau_{n}, X^{n}\left(\tau^{n}\right)\right)\right) \mathbf{1}_{\tau_{n} \leq \theta_{n}}+v\left(\theta_{n}, X^{n}\left(\theta^{n}\right)\right) \mathbf{1}_{\tau_{n}>\theta_{n}}\right]
\end{aligned}
$$

Since $\left(\varphi\left(t_{n}, x_{n}\right)-v\left(t_{n}, x_{n}\right)\right) \rightarrow 0$ and $0 \in \tilde{K}$, this leads to a contradiction to (2.13) for $n$ large enough.

2. We now prove that $v_{*}\left(t_{0}, x_{0}\right) \geq g\left(t_{0}, x_{0}\right)$ for all $\left(t_{0}, x_{0}\right) \in \partial_{x} D^{*}$. To see this, we assume to the contrary that

$$
v_{*}\left(t_{0}, x_{0}\right)<g\left(t_{0}, x_{0}\right)
$$

for some $\left(t_{0}, x_{0}\right) \in \partial_{x} D^{*}$ and work toward a contradiction to (6.3). Let $\varphi \in C^{2}\left(\bar{D}^{*}\right)$ be such that

$$
0=\left(v_{*}-\varphi\right)\left(t_{0}, x_{0}\right)=\min _{\bar{D}^{*}}(\text { strict })\left(v_{*}-\varphi\right)
$$


For $\varepsilon>0$, define $\phi^{\varepsilon}$ on $\bar{D}^{*}$ by

$$
\phi^{\varepsilon}(t, x)=\varphi(t, x)-\left(d(x)-\frac{d(x)^{2}}{\varepsilon}\right),
$$

where $d$ is defined in $\mathbf{H}_{\mathcal{O}}$. Since $d(x)-\frac{d(x)^{2}}{\varepsilon}>0$ when $0<d(x)<\varepsilon$, it follows that $\left(t_{0}, x_{0}\right)$ is a strict local minimum of $\left(v_{*}-\phi^{\varepsilon}\right)$ for each $\varepsilon>0$. By (6.3) and (6.6), we must therefore have

$$
-\mathcal{L} \varphi\left(t_{0}, x_{0}\right)+\operatorname{Tr}\left[a\left(t_{0}, x_{0}\right) D^{2} d\left(x_{0}\right)\right]-\frac{1}{\varepsilon} \operatorname{Tr}\left[a\left(t_{0}, x_{0}\right) \operatorname{Dd}\left(x_{0}\right) \operatorname{Dd}\left(x_{0}\right)^{\prime}\right] \geq 0,
$$

which leads to a contradiction to (2.2) when $\varepsilon$ tends to 0 .

3. In view of 2 . and the definition of $\hat{g}$ in (3.5), (3.11) concludes the proof.

Proposition 6.4 Assume that $\mathbf{H}_{\tilde{K}}-\mathbf{H}_{g}$ holds. Then, $v_{*}(T, \cdot) \geq \hat{g}(T, \cdot)$ on $\overline{\mathcal{O}}^{*}$.

Proof. Fix $x_{0} \in \overline{\mathcal{O}}^{*}$ and let $\left(t_{n}, x_{n}\right)_{n}$ be a sequence in $D$ converging to $\left(T, x_{0}\right)$ such that $v\left(t_{n}, x_{n}\right) \rightarrow v_{*}\left(T, x_{0}\right)$. Set $\left(\tau_{n}, X^{n}\right)=\left(\tau_{t_{n}, x_{n}}, X_{t_{n}, x_{n}}\right)$. Since $\sigma$ is bounded, see (i) of (2.2), one easily checks that $\left(\tau_{n}, X^{n}\left(\tau_{n}\right)\right) \rightarrow\left(T, x_{0}\right) \mathbb{P}-$ a.s., after possibly passing to a subsequence. In view of $\mathbf{H}_{g}$, it follows that

$$
\liminf _{n \rightarrow \infty}\left(g\left(\tau_{n}, X^{n}\left(\tau_{n}\right)\right) \mathbf{1}_{\tau_{n}<T}+g\left(T, X^{n}(T)\right) \mathbf{1}_{\tau_{n}=T}\right) \geq g\left(T, x_{0}\right) .
$$

Since $g \geq 0$ by assumption and $0 \in \tilde{\mathcal{K}}$, it follows from Fatou's Lemma and (2.11) that $v_{*}\left(T, x_{0}\right) \geq g\left(T, x_{0}\right)$. The proof is concluded by using (3.11) and recalling the definition of $\hat{g}(T, \cdot)$ in $(3.3)$.

\subsection{Subsolution property}

In view of Proposition 6.1, 6.2, 6.3 and 6.4, we already know that $v_{*}$ is a supersolution of $\mathcal{B}_{\mathrm{d}} \varphi=0$ on $\bar{D}^{*}$. To conclude the proof of (i) of Theorem 3.1, it remains to show that $v^{*}$ is a subsolution of $\mathcal{B} \varphi=0$ on $\bar{D}^{*}$. This is a consequence of Proposition 6.5, 6.6 and 6.7 below.

Proposition 6.5 Let $\left(t_{0}, x_{0}\right) \in D$ and $\varphi \in C^{2}\left(\bar{D}^{*}\right)$ be such that $\left(t_{0}, x_{0}\right)$ is a local maximum on $\bar{D}^{*}$ of $v^{*}-\varphi$ satisfying $\left(v^{*}-\varphi\right)\left(t_{0}, x_{0}\right)=0$. Then,

$$
\min \left\{-\mathcal{L} \varphi\left(t_{0}, x_{0}\right) ; \mathcal{H} \varphi\left(t_{0}, x_{0}\right)\right\} \leq 0 \text {. }
$$


Proof. The proof is standard. We assume that

$$
G\left(t_{0}, x_{0}\right):=\inf _{\rho \in \tilde{K}}\left\{-\mathcal{L} \varphi\left(t_{0}, x_{0}\right)+\delta(\rho) \varphi\left(t_{0}, x_{0}\right)-\rho^{\prime} \operatorname{diag}\left[x_{0}\right] D \varphi\left(t_{0}, x_{0}\right)\right\}>0,
$$

and work towards a contradiction. If (6.8) holds, then it follows from (i) of (2.2) that there exists some $\alpha>0$ such that

$$
G(t, x)>0 \quad \text { for all }(t, x) \in B_{0}:=B\left(t_{0}, \alpha\right) \times B\left(x_{0}, \alpha\right) \subset D
$$

Let $\left(t_{n}, x_{n}\right)_{n \geq 0}$ be a sequence in $B_{0}$ such that $\left(t_{n}, x_{n}\right) \rightarrow\left(t_{0}, x_{0}\right)$ and $v\left(t_{n}, x_{n}\right) \rightarrow$ $v^{*}\left(t_{0}, x_{0}\right)$. Observe that $\beta_{n}:=\varphi\left(t_{n}, x_{n}\right)-v\left(t_{n}, x_{n}\right) \rightarrow 0$. Set $X^{n}=X_{t_{n}, x_{n}}$ and define the stopping times

$$
\theta_{n}:=T \wedge \inf \left\{s \in\left[t_{n}, T\right]:\left(s, X^{n}(s)\right) \notin B_{0}\right\}
$$

Let $\partial_{p} B_{0}=\left[t_{0}, t_{0}+\alpha\right] \times \partial B\left(x_{0}, \alpha\right) \cup\left\{t_{0}+\alpha\right\} \times \bar{B}\left(x_{0}, \alpha\right)$ denote the parabolic boundary of $B_{0}$ and observe that

$$
0>-\zeta:=\sup _{(t, x) \in \partial_{p} B_{0}}\left(v^{*}-\varphi\right)(t, x) .
$$

Then, we deduce from Itô's Lemma applied on $\varphi$, (6.9), Girsanov's Theorem, see the discussion in Section 2.2, and the above assertion that

$$
v\left(t_{n}, x_{n}\right)+\beta_{n} \geq \zeta+\sup _{\vartheta \in \tilde{\mathcal{K}}} \mathbb{E}^{\vartheta}\left[\mathcal{E}_{\theta_{n}}^{\vartheta} v\left(\theta_{n}, X^{n}\left(\theta_{n}\right)\right)\right]
$$

Since by construction $\theta_{n}<\tau_{t_{n}, x_{n}}$ and $\beta_{n} \rightarrow 0$, we obtain a contradiction to (2.13).

Proposition 6.6 Assume that $\mathbf{H}_{\mathcal{O}}-\mathbf{H}_{g}$ holds. Let $\left(t_{0}, x_{0}\right) \in \partial_{x} D^{*}$ and $\varphi \in C^{2}\left(\bar{D}^{*}\right)$ be such that $\left(t_{0}, x_{0}\right)$ is a local maximum on $\bar{D}^{*}$ of $v_{*}-\varphi$ satisfying $\left(v_{*}-\varphi\right)\left(t_{0}, x_{0}\right)=0$. Then,

$$
\min \left\{v^{*}\left(t_{0}, x_{0}\right)-\hat{g}\left(t_{0}, x_{0}\right) ; \mathcal{H} \varphi\left(t_{0}, x_{0}\right)\right\} \leq 0
$$

Proof. 1. By using similar arguments as in the proof of Proposition 6.5, we first obtain that

$$
\min \left\{v^{*}\left(t_{0}, x_{0}\right)-g\left(t_{0}, x_{0}\right) ;-\mathcal{L} \varphi\left(t_{0}, x_{0}\right) ; \mathcal{H} \varphi\left(t_{0}, x_{0}\right)\right\} \leq 0
$$


2. We now proceed by contradiction as in 2. of the proof of Proposition 6.3 to show that

$$
\min \left\{v^{*}\left(t_{0}, x_{0}\right)-g\left(t_{0}, x_{0}\right) ; \mathcal{H} \varphi\left(t_{0}, x_{0}\right)\right\} \leq 0
$$

As usual, we can assume that $\left(t_{0}, x_{0}\right)$ is a strict local maximum of $v^{*}-\varphi$ on $\bar{D}^{*}$. Assume that for some $\eta>0$,

$$
\min \left\{v^{*}\left(t_{0}, x_{0}\right)-g\left(t_{0}, x_{0}\right) ; \inf _{\rho \in \tilde{K}_{1}} \delta(\rho) v^{*}\left(t_{0}, x_{0}\right)-\rho^{\prime} \operatorname{diag}\left[x_{0}\right] D \varphi\left(t_{0}, x_{0}\right)\right\}>\eta .
$$

Let $\lambda>0$ be a fixed parameter to be chosen later and for $\varepsilon>0$ set on $\bar{D}^{*}$

$$
\phi^{\varepsilon}(t, x)=\varphi(t, x)+\lambda d(x)-\frac{d^{2}(x)}{\varepsilon}
$$

where $d$ is defined in $\mathbf{H}_{\mathcal{O}}$. For $x \in \bar{O}^{*}$ such that $d(x)<\varepsilon \lambda$ we have $\lambda d(x)-\frac{d^{2}(x)}{\varepsilon} \geq 0$. It follows that $\left(t_{0}, x_{0}\right)$ is a local maximum of $v^{*}-\phi^{\varepsilon}$. Moreover,

$$
\min \left\{v^{*}\left(t_{0}, x_{0}\right)-g\left(t_{0}, x_{0}\right) ; \inf _{\rho \in \tilde{K}_{1}} \delta(\rho) v^{*}\left(t_{0}, x_{0}\right)-\rho^{\prime} \operatorname{diag}\left[x_{0}\right] D \phi^{\varepsilon}\left(t_{0}, x_{0}\right)\right\}>0,
$$

for $\lambda>0$ small enough since $d\left(x_{0}\right)=0$ and therefore $D \phi^{\varepsilon}\left(t_{0}, x_{0}\right)=D \varphi\left(t_{0}, x_{0}\right)+$ $\lambda D d\left(x_{0}\right)-2 D d\left(x_{0}\right) d\left(x_{0}\right) / \varepsilon=D \varphi\left(t_{0}, x_{0}\right)+\lambda D d\left(x_{0}\right)$. Thus, it follows from 1 . that we must have

$$
-\mathcal{L}\left(\varphi\left(t_{0}, x_{0}\right)+\lambda d\left(x_{0}\right)\right)+\frac{1}{\varepsilon} \operatorname{Tr}\left[a\left(t_{0}, x_{0}\right) \operatorname{Dd}\left(x_{0}\right) \operatorname{Dd}\left(x_{0}\right)^{\prime}\right] \leq 0 .
$$

Sending $\varepsilon \rightarrow 0$ leads to a contradiction to (i) of (2.2).

Proposition 6.7 Assume that $\mathbf{H}_{g}$ holds. Then, $v^{*}(T, \cdot) \leq \hat{g}(T, \cdot)$ on $\overline{\mathcal{O}}^{*}$.

Proof. 1. Let $\left(t_{n}, x_{n}\right)_{n}$ be a sequence in $D$ which converges to $\left(T, x_{0}\right)$ and such that $v\left(t_{n}, x_{n}\right) \rightarrow v^{*}\left(T, x_{0}\right)$. Set $\left(\tau_{n}, X^{n}\right)=\left(\tau_{t_{n}, x_{n}}, X_{t_{n}, x_{n}}\right)$. By the dual formulation (2.11), there is some $\vartheta^{n} \in \tilde{\mathcal{K}}$ such that

$$
v\left(t_{n}, x_{n}\right) \leq \mathbb{E}^{\vartheta^{n}}\left[e^{-\int_{t_{n}}^{\tau_{n}} \delta\left(\vartheta_{s}^{n}\right) d s} g\left(\tau_{n}, X^{n}\left(\tau_{n}\right)\right)\right]+n^{-1}
$$

Since $\tilde{K}$ is a convex cone, $\delta$ is sublinear and $g \geq 0$, it follows that

$$
e^{-\int_{t_{n}}^{\tau_{n}} \delta\left(\vartheta_{s}^{n}\right) d s} g\left(\tau_{n}, X^{n}\left(\tau_{n}\right)\right) \leq e^{-\delta\left(\int_{t_{n}}^{\tau_{n}} \vartheta_{s}^{n} d s\right)} g\left(\tau_{n}, X^{n}\left(\tau_{n}\right)\right) \leq \sup _{t_{n} \leq t \leq T} \hat{g}\left(t, X^{n}(t) e^{-\int_{t_{n}}^{t} \vartheta_{s}^{n} d s}\right)
$$


by definition of $\hat{g}$ in (3.3)-(3.5). In view of the above inequalities and the definition of $\left(t_{n}, x_{n}\right)$, it remains to show that

$$
\limsup _{n \rightarrow \infty} \mathbb{E}^{\vartheta^{n}}\left[\sup _{t_{n} \leq t \leq T} \hat{g}\left(t, Z^{n}(t)\right)\right] \leq \hat{g}\left(T, x_{0}\right),
$$

where $Z^{n}:=X^{n} e^{-\int_{t_{n}}^{\cdot} \vartheta_{s}^{n} d s}$ solves on $\left[t_{n}, T\right]$

$$
d Z^{n}(t)=\operatorname{diag}\left[Z^{n}(t)\right] \sigma\left(t, X^{n}(t)\right) d W_{t}^{n}, Z^{n}\left(t_{n}\right)=x_{n},
$$

and $W^{n}$ is a standard Brownian motion under $\mathbb{Q}^{\vartheta^{n}}$, recall the discussion of Section 2.2. Using the boundedness assumption on $\sigma$, see (2.2), we deduce from standard arguments that there is a constant $C>0$ independent of $n$ such that

$$
\mathbb{E}^{\vartheta^{n}}\left[\sup _{t_{n} \leq t \leq T}\left|Z^{n}(t)-x_{0}\right|\right] \leq C\left(\left|x_{n}-x_{0}\right|+\left(T-t_{n}\right)^{1 / 2}\right)
$$

We shall prove in 2. that, for each $\varepsilon>0$, there is a Lipschitz function $\Psi_{\varepsilon}$ such that $\left|\hat{g}\left(T, x_{0}\right)-\Psi_{\varepsilon}\left(T, x_{0}\right)\right| \leq \varepsilon$ and $\Psi_{\varepsilon} \geq \hat{g}$. It follows that, for each $\varepsilon$, we can find some finite $K_{\varepsilon}>0$ such that

$$
\begin{aligned}
\limsup _{n \rightarrow \infty} \mathbb{E}^{\vartheta^{n}}\left[\sup _{t_{n} \leq t \leq T} \hat{g}\left(t, Z^{n}(t)\right)\right] & \leq \limsup _{n \rightarrow \infty} \mathbb{E}^{\vartheta^{n}}\left[\sup _{t_{n} \leq t \leq T} \Psi_{\varepsilon}\left(t, Z^{n}(t)\right)\right] \\
& \leq \Psi_{\varepsilon}\left(T, x_{0}\right)+\limsup _{n \rightarrow \infty} K_{\varepsilon}\left(\left|x_{n}-x_{0}\right|+\left(T-t_{n}\right)^{1 / 2}\right) \\
& =\Psi_{\varepsilon}\left(T, x_{0}\right) .
\end{aligned}
$$

By definition of $\Psi_{\varepsilon}$ this implies that

$$
\limsup _{n \rightarrow \infty} \mathbb{E}^{\vartheta^{n}}\left[\sup _{t_{n} \leq t \leq T} \hat{g}\left(t, Z^{n}(t)\right)\right] \leq \hat{g}\left(T, x_{0}\right)+\varepsilon,
$$

and the proof of (6.11) is concluded by sending $\varepsilon$ to 0 .

2. We conclude this proof by constructing the sequence of functions $\left(\Psi_{\varepsilon}\right)_{\varepsilon>0}$. For $(t, x) \in[0, T] \times(0, \infty)^{d}$, we define

$$
G_{k}(t, x)=\sup _{(s, z) \in[0, T] \times(0, \infty)^{d}}[\hat{g}(s, z)-k(|s-t|+|z-x|)], k \geq 1 .
$$

Clearly, $G_{k} \geq \hat{g}$ and $G_{k}$ is $k$-Lipschitz. Moreover, taking $k$ large enough, it follows from the linear growth and upper-semicontinuity assumptions on $\hat{g}$, see $\mathbf{H}_{g}$, that, for 
all $(t, x) \in[0, T] \times(0, \infty)^{d}$, the maximum is attained in the above definition by some $\left(t_{k}(t, x), x_{k}(t, x)\right)$. In particular,

$$
G_{k}(t, x)=\hat{g}\left(t_{k}(t, x), x_{k}(t, x)\right)-k\left(\left|t_{k}(t, x)-t\right|+\left|x_{k}(t, x)-x\right|\right) \geq \hat{g}(t, x) .
$$

Using the linear growth of $\hat{g}$ again, we deduce that $\left(t_{k}(t, x), x_{k}(t, x)\right) \rightarrow(t, x)$ as $k \rightarrow \infty$ after possibly passing to a subsequence. Since $\hat{g}$ is upper-semicontinuous, this also implies that

$$
\hat{g}\left(T, x_{0}\right) \geq \limsup _{k \rightarrow \infty} \hat{g}\left(t_{k}\left(T, x_{0}\right), x_{k}\left(T, x_{0}\right)\right) \geq \limsup _{k \rightarrow \infty} G_{k}\left(T, x_{0}\right) \geq \hat{g}\left(T, x_{0}\right) .
$$

We can then choose $k_{\varepsilon}$ such that $\left|G_{k_{\varepsilon}}\left(T, x_{0}\right)-\hat{g}\left(T, x_{0}\right)\right| \leq \varepsilon$ and set $\Psi_{\varepsilon}:=G_{k_{\varepsilon}}$.

\subsection{Characterization of $v_{*}$ as the smallest supersolution}

In this section, we prove that $v=v_{*}$ on $D$ and that $v_{*}$ is the smallest supersolution of (3.8).

To this end, we introduce a sequence of approximating control problems as follows. For all $\eta \geq 1$, we define $\tilde{K}_{\eta}$ as the set of elements $\rho \in \tilde{K}$ such that $|\rho| \leq \eta$ and $\tilde{\mathcal{K}}_{\eta}$ as the set of elements $\vartheta \in \tilde{\mathcal{K}}$ that take values in $\tilde{K}_{\eta}$. We then define on $\bar{D}^{*}$

$$
w_{\eta}(t, x)=\sup _{\vartheta \in \tilde{\mathcal{K}}_{\eta}} \mathbb{E}^{\vartheta}\left[\mathcal{E}_{\tau}^{\vartheta} g\left(\tau_{t, x}, X_{t, x}\left(\tau_{t, x}\right)\right)\right] .
$$

It is clear that $w_{\eta}$ is a non-decreasing sequence and it follows directly from Theorem 2.1 and the definition of $\tilde{\mathcal{K}}$ that

$$
\lim _{\eta \rightarrow \infty} \uparrow w_{\eta}(t, x)=v(t, x) \text { for all }(t, x) \in \bar{D}^{*} .
$$

For $\eta \geq 1$, let us introduce the operator $\mathcal{G}_{\eta}$ defined for smooth functions by

$$
\mathcal{G}_{\eta} \varphi(t, x):=\min _{\rho \in \tilde{K}_{\eta}}\left\{-\mathcal{L} \varphi(t, x)+\delta(\rho) \varphi(t, x)-\rho^{\prime} \operatorname{diag}[x] D \varphi(t, x)\right\}
$$

Proposition 6.8 Let the conditions $\mathbf{H}_{g}-\mathbf{H}_{\mathcal{O}}$ hold. Then, for all $\eta \geq 1$, w $w_{\eta}^{*}$ is a viscosity subsolution on $D$ of

$$
\mathcal{G}_{\eta} \varphi\left(t_{0}, x_{0}\right)=0
$$

Moreover, $w_{\eta}^{*} \leq \hat{g}$ on $\partial_{x} D^{*} \cup \partial_{T} D^{*}$. 
Proof. The proof is standard. Set $\varphi \in C^{2}(D)$ and let $\left(t_{0}, x_{0}\right)$ be a strict global maximizer of $w_{\eta}^{*}-\varphi$ on $\bar{D}^{*}$ such that $\left(w_{\eta}^{*}-\varphi\right)\left(t_{0}, x_{0}\right)=0$.

1. If $\left(t_{0}, x_{0}\right) \in D$ then the result follows from the same arguments as in the proof of Proposition 6.5.

2. Arguing as in Proposition 6.5 again, we deduce that

$$
\min \left\{w_{\eta}^{*}\left(t_{0}, x_{0}\right)-\hat{g}\left(t_{0}, x_{0}\right), \mathcal{G}_{\eta} \varphi\left(t_{0}, x_{0}\right)\right\} \leq 0
$$

if $\left(t_{0}, x_{0}\right) \in \partial_{x} D^{*}$. The required result is then obtained by arguing as in 2 . of the proof of Proposition 6.6.

3. Since $w_{\eta}^{*} \leq v^{*}$, the inequality $w_{\eta}^{*}(T, \cdot) \leq \hat{g}(T, \cdot)$ follows from Proposition 6.7.

Proposition 6.9 Assume that $\mathbf{H}_{g}$ hold. Let $u$ (resp. w) be a viscosity subsolution (resp. supersolution) of (6.14) on D satisfying the growth condition (3.10). If $u \leq w$ on $\partial_{x} D^{*} \cup \partial_{T} D^{*}$, then $u \leq w$ on $\bar{D}^{*}$.

Proof. 1. Given $\kappa>0$, we set $\tilde{u}(t, x)=e^{\kappa t} u(t, x)$ and $\tilde{w}(t, x)=e^{\kappa t} w(t, x)$ so that $\tilde{u}$ and $\tilde{v}$ are respectively sub- and supersolutions of

$$
\tilde{\mathcal{G}}_{\eta} \varphi(t, x):=\min _{\rho \in \tilde{K}_{\eta}}\left\{(\kappa+\delta(\rho)) \varphi(t, x)-\mathcal{L}^{\rho} \varphi(t, x)\right\}=0
$$

where for $\rho \in \tilde{K}$

$$
\mathcal{L}^{\rho} \varphi(t, x):=\mathcal{L} \varphi(t, x)+\rho^{\prime} \operatorname{diag}[x] D \varphi(t, x)
$$

Recall the definition of $\bar{\gamma}$ in $\mathbf{H}_{g}$ and set

$$
\gamma=2 \bar{\gamma} \in \mathbb{R}_{+}^{d} \quad, \quad \tilde{\gamma}=(2, \ldots, 2) \in(0, \infty)^{d} .
$$

Define on $\bar{D}^{*}$

$$
\beta(t, x):=e^{\tau(T-t)}\left(1+x^{\gamma}+x^{\tilde{\gamma}}\right)
$$

Observing that

$$
\begin{aligned}
& \frac{\partial}{\partial t} \beta(t, x)=-\tau \beta(t, x), \operatorname{diag}[x] D \beta(t, x)=e^{\tau(T-t)}\left(x^{\gamma} \gamma+x^{\tilde{\gamma}} \tilde{\gamma}\right) \\
& \operatorname{Tr}\left[a(t, x) D^{2} \beta(t, x)\right]=e^{\tau(T-t)}\left(x^{\gamma} \operatorname{Tr}\left[\sigma \sigma^{\prime}(t, x) M\right]+x^{\tilde{\gamma}} \operatorname{Tr}\left[\sigma \sigma^{\prime}(t, x) \tilde{M}\right]\right)
\end{aligned}
$$


with $M:=\left[\gamma^{i}\left(\gamma^{i}-1\right) \mathbf{1}_{i=j}+\gamma^{i} \gamma^{j} \mathbf{1}_{i \neq j}\right]_{i j}$ and $\tilde{M}$ defined similarly, it follows from (i) of (2.2) and the compactness of $\tilde{K}_{\eta}$ that we can find $\tau>0$ such that

$$
\tilde{\mathcal{G}}_{\eta} \beta(t, x) \geq 0 \quad \text { on } \bar{D}^{*} \text {. }
$$

2. We now argue by contradiction and assume that

$$
\sup _{\bar{D}^{*}}(\tilde{u}-\tilde{w})>0
$$

2.1. In view of the growth condition on $\tilde{u}, \tilde{w}$ and (6.15), we then have

$$
0<2 m:=\sup _{\bar{D}^{*}}(\tilde{u}-\tilde{w}-2 \alpha \beta)<\infty
$$

for $\alpha>0$ small enough. For $x \in \bar{D}^{*}$, set

$$
f(x)=\sum_{i=1}^{d}\left(x^{i}\right)^{-2}
$$

Combining the growth condition on $\tilde{u}, \tilde{w}$ with (6.15) and the definition of $f$ implies that, for each $\varepsilon>0$, the upper-semicontinuous function

$$
\Phi^{\varepsilon}:=\tilde{u}-\tilde{w}-2(\alpha \beta+\varepsilon f)
$$

admits a maximum $\left(t_{\varepsilon}, x_{\varepsilon}\right)$ on $\bar{D}^{*}$. By $(6.17)$, we can choose $\varepsilon$ small enough so that

$$
\Phi^{\varepsilon}\left(t_{\varepsilon}, x_{\varepsilon}\right) \geq m>0
$$

Let $\left(t_{0}^{\varepsilon}, x_{0}^{\varepsilon}\right)$ be a sequence in $D$ such that $\Phi^{\varepsilon}\left(t_{0}^{\varepsilon}, x_{0}^{\varepsilon}\right) \rightarrow 2 m$. By $(6.17)$ and definition of $\left(t_{\varepsilon}, x_{\varepsilon}\right)$, we have

$$
\begin{aligned}
\liminf _{\varepsilon \rightarrow 0}\left(2 m-2 \varepsilon f\left(x_{\varepsilon}\right)\right) & \geq \liminf _{\varepsilon \rightarrow 0}(\tilde{u}-\tilde{w}-2(\alpha \beta+\varepsilon f))\left(t_{\varepsilon}, x_{\varepsilon}\right) \\
& \geq \lim _{\varepsilon \rightarrow 0}(\tilde{u}-\tilde{w}-2(\alpha \beta+\varepsilon f))\left(t_{0}^{\varepsilon}, x_{0}^{\varepsilon}\right) \\
& =2 m .
\end{aligned}
$$

This shows that

$$
\limsup _{\varepsilon \rightarrow 0} \varepsilon f\left(x_{\varepsilon}\right)=\limsup _{\varepsilon \rightarrow 0} \varepsilon \sum_{i=1}^{d}\left(x_{\varepsilon}^{i}\right)^{-2}=0,
$$


which, by (i) of (2.2) and the compactness of $\tilde{K}_{\eta}$, implies

$$
\limsup _{\varepsilon \rightarrow 0} \sup _{\rho \in \tilde{K}_{\eta}} \varepsilon\left(\left|f\left(x_{\varepsilon}\right)\right|+\left|\mathcal{L}^{\rho} f\left(x_{\varepsilon}\right)\right|\right)=0 .
$$

2.2. For $(t, x) \in[0, T] \times \mathbb{R}^{d}$, set $G^{\varepsilon}(t, x)=\left|t-t_{\varepsilon}\right|^{4}+\left|x-x_{\varepsilon}\right|^{4}$. Given $n>0$, it follows from similar arguments as above that the map

$$
\begin{aligned}
\Phi_{n}^{\varepsilon}(t, x, y) & :=\tilde{u}(t, x)-\tilde{w}(t, y)-\frac{n}{2}|x-y|^{2}-\alpha(\beta(t, x)+\beta(t, y)) \\
& -\varepsilon\left(f(x)+f(y)+G^{\varepsilon}(t, x)\right),
\end{aligned}
$$

also admits a maximum point $\left(t_{n}^{\varepsilon}, x_{n}^{\varepsilon}, y_{n}^{\varepsilon}\right) \in \bar{D}^{*}$ which necessarily satisfies

$$
\Phi_{n}^{\varepsilon}\left(t_{n}^{\varepsilon}, x_{n}^{\varepsilon}, y_{n}^{\varepsilon}\right) \geq \Phi_{n}^{\varepsilon}\left(t_{\varepsilon}, x_{\varepsilon}, x_{\varepsilon}\right)=\Phi^{\varepsilon}\left(t_{\varepsilon}, x_{\varepsilon}\right) \geq m>0
$$

Using the growth assumption on $u$ and $w,(6.15)$ and the definition of $f$ again, one easily checks that this implies that the sequence $\left(t_{n}^{\varepsilon}, x_{n}^{\varepsilon}, y_{n}^{\varepsilon}\right)_{n}$ is bounded and therefore converges, after possibly passing to a subsequence. Moreover, (6.21) implies that $n \mid x_{n}^{\varepsilon}-$ $\left.y_{n}^{\varepsilon}\right|^{2}+\varepsilon f\left(x_{n}^{\varepsilon}\right)$ is bounded. Thus, there is $\left(\bar{t}_{\varepsilon}, \bar{x}_{\varepsilon}\right) \in \bar{D}^{*}$ such that $\left(t_{n}^{\varepsilon}, x_{n}^{\varepsilon}, y_{n}^{\varepsilon}\right) \rightarrow\left(\bar{t}_{\varepsilon}, \bar{x}_{\varepsilon}, \bar{x}_{\varepsilon}\right)$ and, by definition of $\left(t_{\varepsilon}, x_{\varepsilon}\right)$ and (6.21), we must have

$$
\begin{aligned}
\Phi^{\varepsilon}\left(t_{\varepsilon}, x_{\varepsilon}\right) & \geq \Phi^{\varepsilon}\left(\bar{t}_{\varepsilon}, \bar{x}_{\varepsilon}\right) \\
& \geq \limsup _{n \rightarrow \infty}\left(\Phi^{\varepsilon}\left(\bar{t}_{\varepsilon}, \bar{x}_{\varepsilon}\right)-\frac{n}{2}\left|x_{n}^{\varepsilon}-y_{n}^{\varepsilon}\right|^{2}-\varepsilon G^{\varepsilon}\left(t_{n}^{\varepsilon}, x_{n}^{\varepsilon}\right)\right) \\
& \geq \Phi^{\varepsilon}\left(t_{\varepsilon}, x_{\varepsilon}\right) .
\end{aligned}
$$

This shows that, up to a subsequence,

$$
\left(t_{n}^{\varepsilon}, x_{n}^{\varepsilon}\right) \rightarrow\left(t_{\varepsilon}, x_{\varepsilon}\right) \in \bar{D}^{*}, \Phi_{n}^{\varepsilon}\left(t_{n}^{\varepsilon}, x_{n}^{\varepsilon}, y_{n}^{\varepsilon}\right) \rightarrow \Phi^{\varepsilon}\left(t_{\varepsilon}, x_{\varepsilon}\right) \quad \text { and } \quad n\left|x_{n}^{\varepsilon}-y_{n}^{\varepsilon}\right|^{2} \rightarrow 0
$$

as $n \rightarrow \infty$.

3. Since the upper-semicontinuous function $u-w$ is non-positive on $\partial_{x} D^{*} \cup \partial_{T} D^{*}$, it follows from $(6.19)$ that $\left(t_{\varepsilon}, x_{\varepsilon}\right) \in D$ and that we may assume that $\left(t_{n}^{\varepsilon}, x_{n}^{\varepsilon}, y_{n}^{\varepsilon}\right) \in$ $[0, T) \times \mathcal{O}^{2}$ for each $n>0$. Using Ishii's Lemma and following standard arguments, see Theorem 8.3 and the discussion after Theorem 3.2 in [4], we deduce from the viscosity property of $\tilde{u}$ and $\tilde{w}$ that for some $\hat{\rho}_{n}^{\varepsilon}$ in the compact set $\tilde{K}_{\eta}$

$$
\begin{aligned}
0 & \leq\left(\kappa+\delta\left(\hat{\rho}_{n}^{\varepsilon}\right)\right)\left(\tilde{w}\left(t_{n}^{\varepsilon}, y_{n}^{\varepsilon}\right)-\tilde{u}\left(t_{n}^{\varepsilon}, x_{n}^{\varepsilon}\right)\right)+\frac{1}{2} \operatorname{Tr}\left[a\left(t_{n}^{\varepsilon}, x_{n}^{\varepsilon}\right) A_{n}^{\varepsilon}-a\left(t_{n}^{\varepsilon}, y_{n}^{\varepsilon}\right) B_{n}^{\varepsilon}\right] \\
& +\left(\hat{\rho}_{n}^{\varepsilon}\right)^{\prime} \operatorname{diag}\left[x_{n}^{\varepsilon}-y_{n}^{\varepsilon}\right] q_{n}^{\varepsilon}+\left\{\mathcal{L}^{\hat{\rho}_{n}^{\varepsilon}}\left(\alpha \beta+\varepsilon\left[f+G^{\varepsilon}\right]\right)\left(t_{n}^{\varepsilon}, x_{n}^{\varepsilon}\right)+\mathcal{L}^{\hat{\rho}_{n}^{\varepsilon}}(\alpha \beta+\varepsilon f)\left(t_{n}^{\varepsilon}, y_{n}^{\varepsilon}\right)\right\}
\end{aligned}
$$


where

$$
q_{n}^{\varepsilon}:=n\left(x_{n}^{\varepsilon}-y_{n}^{\varepsilon}\right)
$$

and $A_{n}^{\varepsilon}, B_{n}^{\varepsilon}$ are two symmetric matrices satisfying

$$
-3 n\left(\begin{array}{cc}
I_{d} & 0 \\
0 & I_{d}
\end{array}\right) \leq\left(\begin{array}{cc}
A_{n}^{\varepsilon} & 0 \\
0 & -B_{n}^{\varepsilon}
\end{array}\right) \leq 3 n\left(\begin{array}{cc}
I_{d} & -I_{d} \\
-I_{d} & I_{d}
\end{array}\right) .
$$

Using (6.16), (6.21), (6.22), (6.23), (6.24) and (i) of (2.2), we then deduce that

$$
\begin{aligned}
0 & \leq-m\left(\kappa+\delta\left(\hat{\rho}_{n}^{\varepsilon}\right)\right)+C n\left|x_{n}^{\varepsilon}-y_{n}^{\varepsilon}\right|^{2}-\varepsilon\left(\kappa+\delta\left(\hat{\rho}_{n}^{\varepsilon}\right)\right)\left\{\left(f+G^{\varepsilon}\right)\left(t_{n}^{\varepsilon}, x_{n}^{\varepsilon}\right)+f\left(y_{n}^{\varepsilon}\right)\right\} \\
& +\varepsilon\left\{\mathcal{L}^{\hat{\rho}_{n}^{\varepsilon}}\left(f+G^{\varepsilon}\right)\left(t_{n}^{\varepsilon}, x_{n}^{\varepsilon}\right)+\mathcal{L}^{\hat{\rho}_{n}^{\varepsilon}} f\left(y_{n}^{\varepsilon}\right)\right\}
\end{aligned}
$$

for some $C>0$ independent of $n$. Sending $n$ to $\infty$, it follows from the compactness of $\tilde{K}_{\eta}$ and (6.22) that

$$
0 \leq-m\left(\kappa+\delta\left(\hat{\rho}^{\varepsilon}\right)\right)+2 \varepsilon\left\{\mathcal{L}^{\hat{\rho}^{\varepsilon}} f\left(x_{\varepsilon}\right)-\left(\kappa+\delta\left(\hat{\rho}^{\varepsilon}\right)\right) f\left(x_{\varepsilon}\right)\right\}
$$

for some $\hat{\rho}^{\varepsilon} \in \tilde{K}_{\eta}$. Sending $\varepsilon$ to 0 and using (6.20) finally leads to a contradiction since $\kappa, m>0$ and $\delta \geq 0$ by (2.9).

We now conclude the proof of Theorem 3.1.

Proof of (ii) and (iii) of Theorem 3.1. Observe that a supersolution $u$ of $\mathcal{B}_{\mathrm{d}} \varphi=0$ on $\bar{D}^{*}$ is also a supersolution of (6.14) on $D$, and, by Proposition 6.8, satisfies $u \geq w_{\eta}$ on $\partial_{x} D^{*} \cup \partial_{T} D^{*}$ for all $\eta \geq 1$. In view of Proposition 6.9 and (6.13), it follows that $u \geq \lim _{\eta \rightarrow \infty} \uparrow w_{\eta}=v$ on $D$ whenever $u$ satisfies (3.10). In particular, since $v_{*}$ is a supersolution of (3.8) satisfying (3.10), see Proposition 3.1, we have $v_{*} \geq v$ so that $v_{*}=v \leq u$ on $D$ and $v_{*} \leq u_{*}$ on $\bar{D}^{*}$.

\section{A uniqueness result}

We now proceed with the proof of Theorem 3.2. It is an immediate consequence of Proposition 3.1, Theorem 3.1 and the following comparison result.

Theorem 7.1 Assume that the conditions of Theorem 3.2 hold. Let $u$ be an uppersemicontinuous viscosity subsolution of $\mathcal{B} \varphi=0$ on $\bar{D}^{*}$. Assume furthermore that $u$ satisfies the growth condition (3.10). Then, $u \leq v_{*}$ on $\bar{D}^{*}$. 
Remark 7.1 1. It will be clear from the proof that the above Theorem can be stated as follows. Let $u$ and $w$ be respectively sub- and supersolution of $\mathcal{B} \varphi=0$ and $\mathcal{B}_{\mathrm{d}} \varphi=0$ on $D \cup \partial_{x} D^{*}$ satisfying the growth condition (3.10). Assume further that $w$ satisfies

C: $\forall(t, x) \in D \cup \partial_{x} D^{*}$ and $\rho \in \tilde{K}_{1}(x, \overline{\mathcal{O}}), \exists \lambda_{0}>0$ s.t. $\lambda \in\left[0, \lambda_{0}\right] \mapsto w\left(t, x e^{\lambda \rho}\right) e^{-\lambda \delta(\rho)}$ is non-increasing.

Then, $u \leq w$ on $\partial_{T} D^{*}$ implies $u \leq w$ on $\bar{D}^{*}$.

2. One can actually show that any supersolution of $\mathcal{H}_{\mathrm{d}} \varphi=0$ satisfies the condition $\mathbf{C}$. Since it is not useful for our main result, we do not provide the proof which is rather long.

3. Combining the above assertions provides a general comparison result for super- and subsolutions of, respectively, $\mathcal{B}_{\mathrm{d}} \varphi=0$ and $\mathcal{B} \varphi=0$ on $D \cup \partial_{x} D^{*}$.

In order to prove Theorem 7.1, we need the following intermediate Lemma.

Lemma 7.1 Assume that $\mathbf{H}_{\mathcal{O}}$ holds. Fix $x_{0} \in \partial \mathcal{O}^{*}$. If $\rho \in \tilde{K}_{1}$ satisfies

$$
\operatorname{Dd}\left(x_{0}\right)^{\prime} \operatorname{diag}\left[x_{0}\right] \rho>0,
$$

then there exists some positive parameters $r_{0}$ and $\lambda_{0}$ such that $x e^{\lambda \rho} \in \mathcal{O}$ for all $x \in$ $B\left(x_{0}, r_{0}\right) \cap \overline{\mathcal{O}}$ and $\lambda \in\left(0, \lambda_{0}\right)$.

Proof. Recall from $\mathbf{H}_{\mathcal{O}}$ that the function $d$ is $C^{2}$ on a neighbourhood of $x_{0}$. Thus, $\operatorname{Dd}\left(x_{0}\right)^{\prime} \operatorname{diag}\left[x_{0}\right] \rho>0$ implies that for some $\delta_{0}, r_{0}>0$

$$
\operatorname{Dd}(\bar{x})^{\prime} \operatorname{diag}[x] \rho \geq \delta_{0} \quad \text { for all } \bar{x}, x \in B\left(x_{0}, r_{0}\right) .
$$

Given that $x e^{\lambda \rho}-x=\lambda \operatorname{diag}[x] \rho+o(\lambda)$, we can fix some $\lambda_{0}>0$ such that, for all $x \in B\left(x_{0}, r_{0} / 2\right)$ and $\lambda \in\left(0, \lambda_{0}\right)$

$$
\left[x, x e^{\lambda \rho}\right] \subset B\left(x_{0}, r_{0}\right) \quad \text { and } \quad\left|x e^{\lambda \rho}-x-\lambda \operatorname{diag}[x] \rho\right|<\frac{\lambda \delta_{0} / 2}{1+\max _{x \in \bar{B}\left(x_{0}, r_{0}\right)}|D d(x)|} .
$$

Let $x$ be in $B\left(x_{0}, r_{0} / 2\right) \cap \overline{\mathcal{O}}$, so that $d(x) \geq 0$. Since $d$ is $C^{1}$, for each $\lambda \in\left(0, \lambda_{0}\right)$ there exists $\bar{x} \in\left[x, x e^{\lambda \rho}\right] \subset B\left(x_{0}, r_{0}\right)$ such that

$$
\begin{aligned}
d\left(x e^{\lambda \rho}\right) & =d(x)+D d(\bar{x})^{\prime}\left(x e^{\lambda \rho}-x\right) \\
& =d(x)+\lambda D d(\bar{x})^{\prime} \operatorname{diag}[x] \rho+D d(\bar{x})^{\prime}\left[x e^{\lambda \rho}-x-\lambda \operatorname{diag}[x] \rho\right] \\
& \geq d(x)+\lambda \frac{\delta_{0}}{2}>0
\end{aligned}
$$


where the last inequality follows from (7.1) and (7.2). This shows that $x e^{\lambda \rho} \in \mathcal{O}$.

Proof of Theorem 7.1: In order to avoid too many complications, we make the proof under the assumption

$$
\begin{aligned}
\mathbf{H}^{\prime \prime}: & \text { (i) } \exists \varrho>1 \text { s.t. } \varrho \bar{\gamma} \in K \cap(0, \infty)^{d}, \\
& \text { (ii) } 0 \in \operatorname{int}(K), \\
& \text { (iii) } \forall x \in \partial \mathcal{O}^{*} \exists \rho \in \tilde{K}_{1} \text { s.t. } D d(x)^{\prime} \operatorname{diag}[x] \rho>0,
\end{aligned}
$$

in place of $\mathbf{H}^{\prime}$. We shall explain in the last step how to adapt this proof when $\overline{\mathcal{O}}$ is bounded but (i) of $\mathbf{H}^{\prime \prime}$ does not hold, or $0 \notin \operatorname{int}(K)$ but $\overline{\mathcal{O}} \cap \partial \mathbb{R}_{+}^{d}=\emptyset$ and $\operatorname{int}(K) \neq \emptyset$.

1. Given some positive parameter $\kappa$, we introduce the functions $\tilde{u}(t, x):=e^{\kappa t} u(t, x)$, $\tilde{v}(t, x):=e^{\kappa t} v_{*}(t, x)$ and $\tilde{g}(t, x):=e^{\kappa t} \hat{g}(t, x)$. One easily checks that the function $\tilde{u}$ (resp. $\tilde{v}$ ) is a viscosity subsolution (resp. supersolution) of $\tilde{\mathcal{B}} \varphi=0$ (resp. $\tilde{\mathcal{B}}_{\mathrm{d}} \varphi=0$ ), where for a smooth function $\varphi$

$$
\begin{aligned}
\tilde{\mathcal{B}} \varphi & =\left\{\begin{array}{lll}
\min \{\tilde{\mathcal{L}} \varphi, \mathcal{H} \varphi\} & \text { on } & D \\
\min \{\varphi-\tilde{g}, \mathcal{H} \varphi\} & \text { on } & \partial_{x} D^{*} \\
\varphi-\tilde{g} & \text { on } & \partial_{T} D^{*}
\end{array}\right. \\
\tilde{\mathcal{B}}_{\mathrm{d}} \varphi & =\left\{\begin{array}{lll}
\tilde{\mathcal{B}} \varphi & \text { on } & D \cup \partial_{T} D^{*} \\
\min \left\{\varphi-\tilde{g}, \mathcal{H}_{\mathrm{d}} \varphi\right\} & \text { on } & \partial_{x} D^{*}
\end{array}\right.
\end{aligned}
$$

and

$$
\tilde{\mathcal{L}} \varphi:=\kappa \varphi-\mathcal{L} \varphi
$$

Let $\varrho \in \mathbb{R}$ be as in $\mathbf{H}^{\prime \prime}$, i.e.

$$
\gamma:=\varrho \bar{\gamma} \in K \cap(0, \infty)^{d} \quad \text { and } \quad \varrho>1 .
$$

Since $0 \in \operatorname{int}(K)$ by $\mathbf{H}^{\prime \prime}$, it follows from (2.9) and Remark 2.4 that the map defined by $\beta(t, x)=e^{\tau(T-t)}\left(1+x^{\gamma}\right)$ on $\bar{D}^{*}$ satisfies

$$
H(\beta(t, x), \operatorname{diag}[x] D \beta(t, x)) \geq c_{K}>0 \quad \text { for all }(t, x) \in \bar{D}^{*} .
$$

Moreover, by the same computations as in the proof of Proposition 6.9, one easily checks that we can choose $\tau$ large enough so that

$$
\tilde{\mathcal{L}} \beta \geq 0 \quad \text { on } \bar{D}^{*} .
$$


2. We argue by contradiction. We assume that

$$
\sup _{\bar{D}^{*}}\left(u-v_{*}\right)>0
$$

and work towards a contradiction.

2.1. In this step, we follow the same construction as in the proof of Proposition 6.9. By the growth condition on $\tilde{u}, \tilde{v}$ and (7.3), we deduce that

$$
0<2 m:=\sup _{\bar{D}^{*}}(\tilde{u}-\tilde{v}-2 \alpha \beta)<\infty
$$

for $\alpha>0$ small enough. Fix $\varepsilon>0$ and let $f$ be defined as in (6.18). Arguing as in the proof of Proposition 6.9, we obtain that

$$
\Phi^{\varepsilon}:=\tilde{u}-\tilde{v}-2(\alpha \beta+\varepsilon f)
$$

admits a maximum $\left(t_{\varepsilon}, x_{\varepsilon}\right)$ on $\bar{D}^{*}$, which, for $\varepsilon>0$ small enough, satisfies

$$
\Phi^{\varepsilon}\left(t_{\varepsilon}, x_{\varepsilon}\right) \geq m>0
$$

Moreover, using the same arguments as in 2.1 of the proof of Proposition 6.9, we obtain that

$$
\limsup _{\varepsilon \rightarrow 0} \sup _{\rho \in \tilde{K}_{1}} \varepsilon\left(\left|f\left(x_{\varepsilon}\right)\right|+\left|\operatorname{diag}\left[x_{\varepsilon}\right] D f\left(x_{\varepsilon}\right)\right|+\left|\mathcal{L} f\left(x_{\varepsilon}\right)\right|\right)=0 .
$$

Finally, since $\beta, f \geq 0$ and $v_{*}(T, \cdot) \geq u(T, \cdot),(7.7)$ implies that $t_{\varepsilon}<T$, i.e.

$$
\left(t_{\varepsilon}, x_{\varepsilon}\right) \in[0, T) \times \overline{\mathcal{O}}^{*}
$$

2.2. In the following, we fix $\rho_{\varepsilon} \in \tilde{K}_{1}$ such that

$$
\begin{array}{ccc}
\rho_{\varepsilon}:=0 & \text { if } & x_{\varepsilon} \in \mathcal{O} \\
D d\left(x_{\varepsilon}\right)^{\prime} \operatorname{diag}\left[x_{\varepsilon}\right] \rho_{\varepsilon}>0 & \text { if } & x_{\varepsilon} \in \partial \mathcal{O}^{*},
\end{array}
$$

see (iii) of $\mathbf{H}^{\prime \prime}$. By Lemma 7.1 and (3.11), we can fix $r_{\varepsilon}, \lambda_{\varepsilon}>0$, such that

$$
x e^{\lambda \rho_{\varepsilon}} \in \mathcal{O} \text { and } e^{-\lambda \delta\left(\rho_{\varepsilon}\right)} \tilde{v}\left(t, x e^{\lambda \rho_{\varepsilon}}\right) \leq \tilde{v}(t, x)
$$

for all $t \in\left(t_{\varepsilon}-r_{\varepsilon}, t_{\varepsilon}+r_{\varepsilon}\right) \cap[0, T), x \in B_{\varepsilon}:=B\left(x_{\varepsilon}, r_{\varepsilon}\right) \cap \overline{\mathcal{O}}$ and $\lambda \in\left(0, \lambda_{\varepsilon}\right)$. 
For $n \geq 1$ and $\zeta \in(0,1)$, we then define the function $\Psi_{n, \zeta}^{\varepsilon}$ on $[0, T] \times\left(\overline{\mathcal{O}}^{*}\right)^{2}$ by $\Psi_{n, \zeta}^{\varepsilon}(t, x, y):=\Theta(t, x, y)-\varepsilon(f(x)+f(y))-\zeta\left(\left|x-x_{\varepsilon}\right|^{2}+\left|t-t_{\varepsilon}\right|^{2}\right)-n^{2}\left|x e^{\frac{\zeta}{n} \rho_{\varepsilon}}-y\right|^{2}$,

where

$$
\Theta(t, x, y):=\tilde{u}(t, x)-\tilde{v}(t, y)-\alpha(\beta(t, x)+\beta(t, y))
$$

It follows from (7.3) and the growth condition (3.10) satisfied by $\tilde{v}$ and $\tilde{u}$ that $\Psi_{n, \zeta}^{\varepsilon}$ attains its maximum at some $\left(t_{n}^{\varepsilon}, x_{n}^{\varepsilon}, y_{n}^{\varepsilon}\right) \in[0, T] \times\left(\overline{\mathcal{O}}^{*}\right)^{2}$. The inequality $\Psi_{n, \zeta}^{\varepsilon}\left(t_{n}^{\varepsilon}, x_{n}^{\varepsilon}, y_{n}^{\varepsilon}\right)$ $\geq \Psi_{n, \zeta}^{\varepsilon}\left(t_{\varepsilon}, x_{\varepsilon}, x_{\varepsilon} e^{\frac{\zeta}{n} \rho_{\varepsilon}}\right)$ implies that

$$
\begin{aligned}
\Theta\left(t_{n}^{\varepsilon}, x_{n}^{\varepsilon}, y_{n}^{\varepsilon}\right) & \geq \Theta\left(t_{\varepsilon}, x_{\varepsilon}, x_{\varepsilon} e^{\frac{\zeta}{n} \rho_{\varepsilon}}\right)-\varepsilon\left(f\left(x_{\varepsilon}\right)+f\left(x_{\varepsilon} e^{\frac{\zeta}{n} \rho_{\varepsilon}}\right)\right) \\
& +n^{2}\left|x_{n}^{\varepsilon} e^{\frac{\zeta}{n} \rho_{\varepsilon}}-y_{n}^{\varepsilon}\right|^{2}+\zeta\left(\left|x_{n}^{\varepsilon}-x^{\varepsilon}\right|^{2}+\left|t_{n}^{\varepsilon}-t_{\varepsilon}\right|^{2}\right)+\varepsilon\left(f\left(x_{n}^{\varepsilon}\right)+f\left(y_{n}^{\varepsilon}\right)\right)
\end{aligned}
$$

which combined with the growth condition (3.10) and (7.3) shows that $n^{2} \mid x_{n}^{\varepsilon} e^{\frac{\zeta}{n} \rho_{\varepsilon}}-$ $\left.y_{n}^{\varepsilon}\right|^{2}+f\left(x_{n}^{\varepsilon}\right)$ is bounded in $n$ so that, up to a subsequence,

$$
\text { (i) } x_{n}^{\varepsilon} e^{\frac{\zeta}{n} \rho_{\varepsilon}}, x_{n}^{\varepsilon}, y_{n}^{\varepsilon} \underset{n \rightarrow \infty}{\longrightarrow} \bar{x}^{\varepsilon} \in \overline{\mathcal{O}}^{*} \text { and } t_{n}^{\varepsilon} \underset{n \rightarrow \infty}{\longrightarrow} \bar{t}^{\varepsilon} \in[0, T] \text {. }
$$

Let $n$ be large enough so that $\frac{\zeta}{n}<\lambda_{\varepsilon}$. Recall from (7.11) that this implies that $\tilde{v}\left(t_{\varepsilon}, x_{\varepsilon} e^{\frac{\zeta}{n} \rho_{\varepsilon}}\right) \leq \tilde{v}\left(t_{\varepsilon}, x_{\varepsilon}\right) e^{\frac{\zeta}{n} \delta\left(\rho_{\varepsilon}\right)}$, which combined with the previous inequality yields

$$
\begin{aligned}
\Theta\left(t_{n}^{\varepsilon}, x_{n}^{\varepsilon}, y_{n}^{\varepsilon}\right) & \geq \tilde{u}\left(t_{\varepsilon}, x_{\varepsilon}\right)-\tilde{v}\left(t_{\varepsilon}, x_{\varepsilon}\right) e^{\frac{\zeta}{n} \delta\left(\rho_{\varepsilon}\right)}-\alpha\left(\beta\left(t_{\varepsilon}, x_{\varepsilon}\right)+\beta\left(t_{\varepsilon}, x_{\varepsilon} e^{\frac{\zeta}{n} \rho_{\varepsilon}}\right)\right) \\
& -\varepsilon\left(f\left(x_{\varepsilon}\right)+f\left(x_{\varepsilon} e^{\frac{\zeta}{n} \rho_{\varepsilon}}\right)\right) \\
& +n^{2}\left|x_{n}^{\varepsilon} e^{\frac{\zeta}{n} \rho_{\varepsilon}}-y_{n}^{\varepsilon}\right|^{2}+\zeta\left(\left|x_{n}^{\varepsilon}-x_{\varepsilon}\right|^{2}+\left|t_{n}^{\varepsilon}-t_{\varepsilon}\right|^{2}\right)+\varepsilon\left(f\left(x_{n}^{\varepsilon}\right)+f\left(y_{n}^{\varepsilon}\right)\right) .
\end{aligned}
$$

Sending $n \rightarrow \infty$ and using the maximum property of $\left(t_{\varepsilon}, x_{\varepsilon}\right)$, we get

$$
\begin{aligned}
0 & \geq \Phi^{\varepsilon}\left(\bar{t}^{\varepsilon}, \bar{x}^{\varepsilon}\right)-\Phi^{\varepsilon}\left(t_{\varepsilon}, x_{\varepsilon}\right) \\
& \geq \limsup _{n \rightarrow \infty}\left(n^{2}\left|x_{n}^{\varepsilon} e^{\frac{\zeta}{n} \rho_{\varepsilon}}-y_{n}^{\varepsilon}\right|^{2}+\zeta\left(\left|x_{n}^{\varepsilon}-x_{\varepsilon}\right|^{2}+\left|t_{n}^{\varepsilon}-t_{\varepsilon}\right|^{2}\right)\right) .
\end{aligned}
$$

Recalling (7.7) and (7.9), this shows that

(ii) $n^{2}\left|x_{n}^{\varepsilon} e^{\frac{\zeta}{n} \rho_{\varepsilon}}-y_{n}^{\varepsilon}\right|^{2}+\zeta\left(\left|x_{n}^{\varepsilon}-x_{\varepsilon}\right|^{2}+\left|t_{n}^{\varepsilon}-t_{\varepsilon}\right|^{2}\right) \underset{n \rightarrow \infty}{\longrightarrow} 0$,

(iii) $\tilde{u}\left(t_{n}^{\varepsilon}, x_{n}^{\varepsilon}\right)-\tilde{v}\left(t_{n}^{\varepsilon}, y_{n}^{\varepsilon}\right) \underset{n \rightarrow \infty}{\longrightarrow}(\tilde{u}-\tilde{v})\left(t_{\varepsilon}, x_{\varepsilon}\right) \geq m+2 \alpha \beta\left(t_{\varepsilon}, x_{\varepsilon}\right)+2 \varepsilon f\left(x_{\varepsilon}\right)$,

(iv) $\left(t_{n}^{\varepsilon}, x_{n}^{\varepsilon}\right) \in[0, T) \times \overline{\mathcal{O}}^{*}$ for $n$ large enough. 
3. From Theorem 8.3 in [4], we deduce that, for each $\eta>0$, there are real coefficients $b_{1, n}^{\varepsilon}, b_{2, n}^{\varepsilon}$ and symmetric matrices $\mathcal{X}_{n}^{\varepsilon, \eta}$ and $\mathcal{Y}_{n}^{\varepsilon, \eta}$ such that

$$
\left(b_{1, n}^{\varepsilon}, p_{n}^{\varepsilon}, \mathcal{X}_{n}^{\varepsilon, \eta}\right) \in \overline{\mathcal{P}}_{\overline{\mathcal{O}}}^{+} \tilde{u}\left(t_{n}^{\varepsilon}, x_{n}^{\varepsilon}\right) \quad \text { and } \quad\left(-b_{2, n}^{\varepsilon}, q_{n}^{\varepsilon}, \mathcal{Y}_{n}^{\varepsilon, \eta}\right) \in \overline{\mathcal{P}}_{\overline{\mathcal{O}}}^{-} \tilde{v}\left(t_{n}^{\varepsilon}, y_{n}^{\varepsilon}\right)
$$

see [4] for the standard notations $\overline{\mathcal{P}}_{\overline{\mathcal{O}}}^{+}$and $\overline{\mathcal{P}}_{\overline{\mathcal{O}}}^{-}$, where

$$
\begin{aligned}
& p_{n}^{\varepsilon}:=2 n^{2}\left(x_{n}^{\varepsilon} e^{\frac{\zeta}{n} \rho_{\varepsilon}}-y_{n}^{\varepsilon}\right) e^{\frac{\zeta}{n} \rho_{\varepsilon}}+2 \zeta\left(x_{n}^{\varepsilon}-x_{\varepsilon}\right)+\alpha D \beta\left(t_{n}^{\varepsilon}, x_{n}^{\varepsilon}\right)+\varepsilon D f\left(x_{n}^{\varepsilon}\right) \\
& q_{n}^{\varepsilon}:=2 n^{2}\left(x_{n}^{\varepsilon} e^{\frac{\zeta}{n} \rho_{\varepsilon}}-y_{n}^{\varepsilon}\right)-\alpha D \beta\left(t_{n}^{\varepsilon}, y_{n}^{\varepsilon}\right)-\varepsilon D f\left(y_{n}^{\varepsilon}\right),
\end{aligned}
$$

and $b_{1, n}^{\varepsilon}, b_{2, n}^{\varepsilon}, \mathcal{X}_{n}^{\varepsilon, \eta}$ and $\mathcal{Y}_{n}^{\varepsilon, \eta}$ satisfy

$$
\left\{\begin{array}{c}
b_{1, n}^{\varepsilon}+b_{2, n}^{\varepsilon}=2 \zeta\left(t_{n}^{\varepsilon}-t_{\varepsilon}\right)-\alpha \tau\left(\beta\left(t_{n}^{\varepsilon}, x_{n}^{\varepsilon}\right)+\beta\left(t_{n}^{\varepsilon}, y_{n}^{\varepsilon}\right)\right) \\
\left(\begin{array}{cc}
\mathcal{X}_{n}^{\varepsilon, \eta} & 0 \\
0 & -\mathcal{Y}_{n}^{\varepsilon, \eta}
\end{array}\right)^{\varepsilon} \leq\left(A_{n}^{\varepsilon}+B_{n}^{\varepsilon}\right)+\eta\left(A_{n}^{\varepsilon}+B_{n}^{\varepsilon}\right)^{2}
\end{array}\right.
$$

with

$$
\begin{aligned}
& A_{n}^{\varepsilon}:=\left(\begin{array}{cc}
2 n^{2} \operatorname{diag}\left[e^{2 \frac{\zeta}{n} \rho_{\varepsilon}}\right]+2 \zeta I_{d} & -2 n^{2} \operatorname{diag}\left[e^{\frac{\zeta}{n} \rho_{\varepsilon}}\right] \\
-2 n^{2} \operatorname{diag}\left[e^{\frac{\zeta}{n} \rho_{\varepsilon}}\right] & 2 n^{2} I_{d}
\end{array}\right) \\
& B_{n}^{\varepsilon}:=\left(\begin{array}{cc}
\alpha D^{2} \beta\left(t_{n}^{\varepsilon}, x_{n}^{\varepsilon}\right)+\varepsilon D^{2} f\left(x_{n}^{\varepsilon}\right) & 0 \\
0 & \alpha D^{2} \beta\left(t_{n}^{\varepsilon}, y_{n}^{\varepsilon}\right)+\varepsilon D^{2} f\left(y_{n}^{\varepsilon}\right)
\end{array}\right) .
\end{aligned}
$$

3.1. We now show that, up to a subsequence,

$$
y_{n}^{\varepsilon} \in \mathcal{O}
$$

In view of (ii), this is clearly true when $x_{\varepsilon} \in \mathcal{O}$. In the case $x_{\varepsilon} \in \partial \mathcal{O}$, we deduce from (ii) that

$$
y_{n}^{\varepsilon}=x_{n}^{\varepsilon} e^{\frac{\zeta}{n} \rho_{\varepsilon}}+o\left(n^{-1}\right)=x_{n}^{\varepsilon}+\frac{\zeta}{n} \operatorname{diag}\left[x_{n}^{\varepsilon}\right] \rho_{\varepsilon}+o\left(n^{-1}\right) .
$$

This implies that, for some $\epsilon_{n} \rightarrow 0$,

$$
d\left(y_{n}^{\varepsilon}\right)=d\left(x_{n}^{\varepsilon}\right)+\frac{\zeta}{n}\left(\operatorname{Dd}\left(x_{n}^{\varepsilon}\right)^{\prime} \operatorname{diag}\left[x_{n}^{\varepsilon}\right] \rho_{\varepsilon}+\epsilon_{n}\right),
$$

so that (7.13) is a consequence of (7.10), the continuity of $D d$ and (ii).

3.2. In this step, we show that there is a subsequence of $\left(t_{n}^{\varepsilon}, x_{n}^{\varepsilon}, y_{n}^{\varepsilon}\right)$ such that

$$
x_{n}^{\varepsilon} \in \mathcal{O} \text { and } \kappa \tilde{u}\left(t_{n}^{\varepsilon}, x_{n}^{\varepsilon}\right)-b_{1, n}^{\varepsilon}-\frac{1}{2} \operatorname{Tr}\left[a\left(t_{n}^{\varepsilon}, x_{n}^{\varepsilon}\right) \mathcal{X}_{n}^{\varepsilon, \eta}\right] \leq 0 .
$$


First observe that we can not have $x_{n}^{\varepsilon} \in \partial \mathcal{O}^{*}$ and $\tilde{u}\left(t_{n}^{\varepsilon}, x_{n}^{\varepsilon}\right) \leq \tilde{g}\left(t_{n}^{\varepsilon}, x_{n}^{\varepsilon}\right)$ for all $n$. In view of (ii), this is obvious if $x_{\varepsilon} \in \mathcal{O}$. If $x_{\varepsilon} \in \partial \mathcal{O}^{*}$, it follows from (7.9) and the viscosity property of $\tilde{v}$ that $\tilde{v}\left(t_{\varepsilon}, x_{\varepsilon}\right) \geq \tilde{g}\left(t_{\varepsilon}, x_{\varepsilon}\right)$. Since $\tilde{g}$ is upper-semicontinuous, see $\mathbf{H}_{g}$, this would imply that $\tilde{u}\left(t_{n}^{\varepsilon}, x_{n}^{\varepsilon}\right) \leq \tilde{v}\left(t_{n}^{\varepsilon}, y_{n}^{\varepsilon}\right)+m / 2$ for $n$ large enough, see (ii), thus leading to a contradiction to (iii) since $\beta, f \geq 0$. By (iv) and the viscosity subsolution property of $\tilde{u}$, we then deduce that either (7.14) holds or

$$
H\left(\tilde{u}\left(t_{n}^{\varepsilon}, x_{n}^{\varepsilon}\right), \operatorname{diag}\left[x_{n}^{\varepsilon}\right] p_{n}^{\varepsilon}\right) \leq 0
$$

Thus, it remains to prove that the above inequality leads to a contradiction. Using the supersolution property of $\tilde{v},(7.13)$, (ii)-(iii) and (2.9), we observe that (7.15) implies

$$
\begin{aligned}
0 & \geq H\left(\tilde{u}\left(t_{n}^{\varepsilon}, x_{n}^{\varepsilon}\right), \operatorname{diag}\left[x_{n}^{\varepsilon}\right] p_{n}^{\varepsilon}\right)-H\left(\tilde{v}\left(t_{n}^{\varepsilon}, y_{n}^{\varepsilon}\right), \operatorname{diag}\left[y_{n}^{\varepsilon}\right] q_{n}^{\varepsilon}\right) \\
& \geq \alpha\left\{H\left(\beta\left(t_{n}^{\varepsilon}, x_{n}^{\varepsilon}\right), \operatorname{diag}\left[x_{n}^{\varepsilon}\right] D \beta\left(t_{n}^{\varepsilon}, x_{n}^{\varepsilon}\right)\right)+H\left(\beta\left(t_{n}^{\varepsilon}, y_{n}^{\varepsilon}\right), \operatorname{diag}\left[y_{n}^{\varepsilon}\right] D \beta\left(t_{n}^{\varepsilon}, y_{n}^{\varepsilon}\right)\right)\right\} \\
& +\varepsilon\left\{H\left(f\left(x_{n}^{\varepsilon}\right), \operatorname{diag}\left[x_{n}^{\varepsilon}\right] D f\left(x_{n}^{\varepsilon}\right)\right)+H\left(f\left(y_{n}^{\varepsilon}\right), \operatorname{diag}\left[y_{n}^{\varepsilon}\right] D f\left(y_{n}^{\varepsilon}\right)\right)\right\} \\
& +\inf _{\rho \in \tilde{K}_{1}} \delta(\rho)\left[\Theta\left(t_{n}^{\varepsilon}, x_{n}^{\varepsilon}, y_{n}^{\varepsilon}\right)-\varepsilon\left(f\left(x_{n}^{\varepsilon}\right)+f\left(y_{n}^{\varepsilon}\right)\right)\right] \\
& -\sup _{\rho \in \tilde{K}_{1}}\left[2 n^{2} \rho^{\prime} \operatorname{diag}\left[x_{n}^{\varepsilon} e^{\frac{\zeta}{n} \rho_{\varepsilon}}-y_{n}^{\varepsilon}\right]\left(x_{n}^{\varepsilon} e^{\frac{\zeta}{n} \rho_{\varepsilon}}-y_{n}^{\varepsilon}\right)+2 \zeta \rho^{\prime} \operatorname{diag}\left[x_{n}^{\varepsilon}\right]\left(x_{n}^{\varepsilon}-x_{\varepsilon}\right)\right] \\
& \geq \inf _{\rho \in \tilde{K}_{1}} \delta(\rho)(m / 2)+2 \alpha H\left(\beta\left(t_{\varepsilon}, x_{\varepsilon}\right), \operatorname{diag}\left[x_{\varepsilon}\right] D \beta\left(t_{\varepsilon}, x_{\varepsilon}\right)\right) \\
& +\epsilon_{n}+\varepsilon\left\{H\left(f\left(x_{\varepsilon}\right), \operatorname{diag}\left[x_{\varepsilon}\right] D f\left(x_{\varepsilon}\right)\right)+H\left(f\left(y_{\varepsilon}\right), \operatorname{diag}\left[y_{\varepsilon}\right] D f\left(y_{\varepsilon}\right)\right)\right\}
\end{aligned}
$$

where $\epsilon_{n} \rightarrow 0$ when $n \rightarrow \infty$, but depend on $\varepsilon$. Recalling (7.4) and (7.8), we get a contradiction for $\varepsilon$ small and $n$ large enough. This concludes the proof of (7.14).

3.3. We can now provide the required contradiction and conclude the proof. Let $\tilde{\sigma}$ be defined on $\bar{D}$ by $\tilde{\sigma}(t, x)=\operatorname{diag}[x] \sigma(t, x)$. By the viscosity supersolution property of $\tilde{v}$, (7.13), (7.14) and (7.12), $\left(t_{n}^{\varepsilon}, x_{n}^{\varepsilon}, y_{n}^{\varepsilon}\right)$ must satisfy

$$
\begin{aligned}
\kappa\left(\tilde{u}\left(t_{n}^{\varepsilon}, x_{n}^{\varepsilon}\right)-\tilde{v}\left(t_{n}^{\varepsilon}, y_{n}^{\varepsilon}\right)\right) & \leq b_{1, n}^{\varepsilon}+b_{2, n}^{\varepsilon}+\frac{1}{2} \operatorname{Tr}\left[a\left(t_{n}^{\varepsilon}, x_{n}^{\varepsilon}\right) \mathcal{X}_{n}^{\varepsilon, \eta}-a\left(t_{n}^{\varepsilon}, y_{n}^{\varepsilon}\right) \mathcal{Y}_{n}^{\varepsilon, \eta}\right] \\
& \leq 2 \zeta\left(t_{n}^{\varepsilon}-t_{\varepsilon}\right)-\alpha \tau\left(\beta\left(t_{n}^{\varepsilon}, x_{n}^{\varepsilon}\right)+\beta\left(t_{n}^{\varepsilon}, y_{n}^{\varepsilon}\right)\right) \\
& +\frac{1}{2} \operatorname{Tr}\left[\Xi\left(t_{n}^{\varepsilon}, x_{n}^{\varepsilon}, y_{n}^{\varepsilon}\right)\left(A_{n}^{\varepsilon}+B_{n}^{\varepsilon}+\eta\left(A_{n}^{\varepsilon}+B_{n}^{\varepsilon}\right)^{2}\right)\right]
\end{aligned}
$$

where

$$
\Xi\left(t_{n}^{\varepsilon}, x_{n}^{\varepsilon}, y_{n}^{\varepsilon}\right):=\left(\begin{array}{cc}
\tilde{\sigma}\left(t_{n}^{\varepsilon}, x_{n}^{\varepsilon}\right) \tilde{\sigma}^{\prime}\left(t_{n}^{\varepsilon}, x_{n}^{\varepsilon}\right) & \tilde{\sigma}\left(t_{n}^{\varepsilon}, y_{n}^{\varepsilon}\right) \tilde{\sigma}^{\prime}\left(t_{n}^{\varepsilon}, x_{n}^{\varepsilon}\right) \\
\tilde{\sigma}\left(t_{n}^{\varepsilon}, x_{n}^{\varepsilon}\right) \tilde{\sigma}^{\prime}\left(t_{n}^{\varepsilon}, y_{n}^{\varepsilon}\right) & \tilde{\sigma}\left(t_{n}^{\varepsilon}, y_{n}^{\varepsilon}\right) \tilde{\sigma}^{\prime}\left(t_{n}^{\varepsilon}, y_{n}^{\varepsilon}\right)
\end{array}\right)
$$


is a non-negative symmetric matrix. Using (ii)-(iii), (7.5) and (7.8), it follows that for $\varepsilon$ small and $n$ large enough

$$
\begin{aligned}
\kappa m / 2 & \leq \kappa\left(\tilde{u}\left(t_{n}^{\varepsilon}, x_{n}^{\varepsilon}\right)-\tilde{v}\left(t_{n}^{\varepsilon}, y_{n}^{\varepsilon}\right)-(\alpha \beta+\varepsilon f)\left(t_{n}^{\varepsilon}, x_{n}^{\varepsilon}\right)-(\alpha \beta+\varepsilon f)\left(t_{n}^{\varepsilon}, y_{n}^{\varepsilon}\right)\right) \\
& \leq 2 \zeta\left(t_{n}^{\varepsilon}-t_{\varepsilon}\right)+\frac{1}{2} \operatorname{Tr}\left[\Xi\left(t_{n}^{\varepsilon}, x_{n}^{\varepsilon}, y_{n}^{\varepsilon}\right)\left(A_{n}^{\varepsilon}+\eta\left(A_{n}^{\varepsilon}+B_{n}^{\varepsilon}\right)^{2}\right)\right]+\theta(\varepsilon, n)
\end{aligned}
$$

where $\theta(\varepsilon, n)$ is independent of $(\eta, \zeta)$ and satisfies

$$
\limsup _{\varepsilon \rightarrow 0} \limsup _{n \rightarrow \infty}|\theta(\varepsilon, n)|=0 .
$$

Sending $\eta \rightarrow 0$ in the previous inequality provides

$$
\kappa m / 2 \leq 2 \zeta\left(t_{n}^{\varepsilon}-t_{\varepsilon}\right)+\frac{1}{2} \operatorname{Tr}\left[\Xi\left(t_{n}^{\varepsilon}, x_{n}^{\varepsilon}, y_{n}^{\varepsilon}\right) A_{n}^{\varepsilon}\right]+\theta(\varepsilon, n),
$$

so that

$$
\begin{aligned}
\kappa m / 2 & \leq 2 \zeta\left(t_{n}^{\varepsilon}-t_{\varepsilon}\right)+\zeta \operatorname{Tr}\left[\tilde{\sigma}\left(t_{n}^{\varepsilon}, x_{n}^{\varepsilon}\right) \tilde{\sigma}^{\prime}\left(t_{n}^{\varepsilon}, x_{n}^{\varepsilon}\right)\right] \\
& +n^{2}\left|\operatorname{diag}\left[x_{n}^{\varepsilon} e^{\frac{\zeta}{n} \rho_{\varepsilon}}\right] \sigma\left(t_{n}^{\varepsilon}, x_{n}^{\varepsilon}\right)-\operatorname{diag}\left[y_{n}^{\varepsilon}\right] \sigma\left(t_{n}^{\varepsilon}, y_{n}^{\varepsilon}\right)\right|^{2}+\theta(\varepsilon, n) .
\end{aligned}
$$

Using (2.2), we now observe that

$$
\begin{aligned}
& \left|\operatorname{diag}\left[x_{n}^{\varepsilon} e^{\frac{\zeta}{n} \rho_{\varepsilon}}\right]\left(\sigma\left(t_{n}^{\varepsilon}, x_{n}^{\varepsilon} e^{\frac{\zeta}{n} \rho_{\varepsilon}}\right)-\sigma\left(t_{n}^{\varepsilon}, x_{n}^{\varepsilon}\right)\right)\right| \\
& \leq\left|\operatorname{diag}\left[x_{n}^{\varepsilon} e^{\frac{\zeta}{n} \rho_{\varepsilon}}\right] \sigma\left(t_{n}^{\varepsilon}, x_{n}^{\varepsilon} e^{\frac{\zeta}{n} \rho_{\varepsilon}}\right)-\operatorname{diag}\left[x_{n}^{\varepsilon}\right] \sigma\left(t_{n}^{\varepsilon}, x_{n}^{\varepsilon}\right)\right|+\left|\operatorname{diag}\left[x_{n}^{\varepsilon} e^{\frac{\zeta}{n} \rho_{\varepsilon}}-x_{n}^{\varepsilon}\right] \sigma\left(t_{n}^{\varepsilon}, x_{n}^{\varepsilon}\right)\right| \\
& \leq C_{\varepsilon}\left|x_{n}^{\varepsilon} e^{\frac{\zeta}{n} \rho_{\varepsilon}}-x_{n}^{\varepsilon}\right| \\
& \leq \zeta C_{\varepsilon} n^{-1}
\end{aligned}
$$

and

$$
\left|\operatorname{diag}\left[x_{n}^{\varepsilon} e^{\frac{\zeta}{n} \rho_{\varepsilon}}\right] \sigma\left(t_{n}^{\varepsilon}, x_{n}^{\varepsilon} e^{\frac{\zeta}{n} \rho_{\varepsilon}}\right)-\operatorname{diag}\left[y_{n}^{\varepsilon}\right] \sigma\left(t_{n}^{\varepsilon}, y_{n}^{\varepsilon}\right)\right|^{2} \leq C_{\varepsilon}\left|x_{n}^{\varepsilon} e^{\frac{\zeta}{n} \rho_{\varepsilon}}-y_{n}^{\varepsilon}\right|^{2}
$$

where $C_{\varepsilon}>0$ denotes a generic constant independent of $n$ and $\zeta$. Plugging this in the previous inequality implies that there is some $C_{\varepsilon}>0$ independent of $n$ and $\zeta$ for which $\kappa m / 2 \leq 2 \zeta\left(t_{n}^{\varepsilon}-t_{\varepsilon}\right)+\zeta \operatorname{Tr}\left[\tilde{\sigma}^{\prime}\left(t_{n}^{\varepsilon}, x_{n}^{\varepsilon}\right) \tilde{\sigma}\left(t_{n}^{\varepsilon}, x_{n}^{\varepsilon}\right)\right]+C_{\varepsilon}\left(\zeta+n^{2}\left|x_{n}^{\varepsilon} e^{\frac{\zeta}{n} \rho_{\varepsilon}}-y_{n}^{\varepsilon}\right|^{2}\right)+\theta(\varepsilon, n)$.

Finally, using (ii) and sending $n$ to $\infty$ and then $\zeta$ to 0 in the last inequality implies

$$
\kappa m / 2 \leq \limsup _{n \rightarrow \infty} \theta(\varepsilon, n)
$$


which by (7.16) provides the required contradiction and concludes the proof.

4. We now explain how to adapt this proof to the alternative assumptions of $\mathbf{H}^{\prime}$.

4.1. Observe that the penalty function $\beta$ is introduced in order to obtain a finite supremum for $\tilde{u}-\tilde{v}-2 \alpha \beta$ and existence of an optimum for $\Phi^{\varepsilon}$ and $\Psi_{n, \zeta}^{\varepsilon}$. If $\overline{\mathcal{O}}$ is bounded, the introduction of such a penalty function is not required and we can reproduce the same proof with $\beta \equiv 0$ whenever $0 \in \operatorname{int}(K)$. Indeed, by Remark 2.4 , inf ${ }_{\rho \in \tilde{K}_{1}} \delta(\rho)>0$ so that we still obtain a contradiction at the end of 3.2. The arguments of 3.3 also work with $\beta \equiv 0$. The case where $0 \notin \operatorname{int}(K)$ is discussed below.

4.2. Similarly, the map $f$ is introduced only to prevent the different maxima to take values outside $\overline{\mathcal{O}}^{*}$. If $\overline{\mathcal{O}} \cap \partial \mathbb{R}_{+}^{d}=\emptyset$, this penalty function is useless and can be fixed to $f \equiv 0$. In this case, one can also fix some $\underline{\gamma} \in \operatorname{int}(K)$, if non-empty, and add the term $e^{\tau(T-t)} x^{\underline{\gamma}}$ in the definition of $\beta$. Thus, $\beta$ becomes $e^{\tau(T-t)}\left(1+x^{\gamma}+x^{\underline{\gamma}}\right)$ or $e^{\tau(T-t)}\left(1+x^{\underline{\gamma}}\right)$ depending whether $\overline{\mathcal{O}}$ is bounded or not, see 4.1 . For fixed $\varepsilon>0$, we deduce from Remark 2.4 and the fact that $\underline{\gamma} \in \operatorname{int}(K)$ that $H(\beta(t, x), \operatorname{diag}[x] D \beta(t, x))$ $>0$. Since $f=0$, there is no $\varepsilon$ to send to 0 at the end of 3.2 and 3.3 , and we obtain the same contradictions by simply sending $n$ to $\infty$ and $\zeta$ to 0 .

\section{References}

[1] Barles G. and P.E. Souganidis (1991). Convergence of approximation schemes for fully nonlinear second order equations. Asymptotic analysis, 4, 271-283.

[2] Bouchard B. and I. Bentahar (2006). Numerical resolution of the barrier option pricing problem under constraints. Forthcoming.

[3] Broadie M., J. Cvitanic̀ and M. Soner (1998). Optimal replication of contingent claims under portfolio constraints. The Review of Financial Studies, 11 (1), 5979 .

[4] Crandall M. G., H. Ishii and P.-L. Lions (1992). User's guide to viscosity solutions of second order Partial Differential Equations. Amer. Math. Soc., 27, 1-67. 
[5] Cvitanic̀ J. and I. Karatzas (1993). Hedging contingent claims with constrained portfolios. Annals of Applied Probability, 3, 652-681.

[6] Cvitanic̀ J. , H. Pham and N. Touzi (1999). Super-replication in stochastic volatility models with portfolio constraints. Journal of Applied Probability, 36, 523-545.

[7] Föllmer H. and D. Kramkov (1997). Optional decomposition under constraints. Probability Theory and Related Fields, 109, 1-25.

[8] Gilbarg D. and N. S. Trudinger (1977). Elliptic partial differential equations of second order. Springer-Verlag.

[9] Karatzas I. and S. E. Shreve (1998). Methods of mathematical finance. SpringerVerlag.

[10] Rockafellar R.T. (1970). Convex Analysis. Princeton University Press, Princeton, NJ.

[11] Shreve S. E., U. Schmock and U. Wystup (2002). Valuation of exotic options under shortselling constraints. Finance and Stochastics, 6, 143-172.

[12] Soner H. M. and N. Touzi (2004). The problem of super-replication under constraints. To appear in Paris-Princeton Lectures in Mathematical Finance, Lecture Notes in Mathematics, Springer-Verlag.

[13] Touzi N. (2000). Direct characterization of the value of super-replication under stochastic volatility and portfolio constraints. Stochastic Processes and their Applications, 88, 305-328. 